Advances in Dynamical Systems and Applications (ADSA).

ISSN 0973-5321, Volume 15, Number 1, (2020) pp. 51-72

(C) Research India Publications

https://dx.doi.org/10.37622/ADSA/15.1.2020.51-72

\title{
Existence of a Solution for a Variational Inequality Associated with the Maxwell-Stokes Type Problem and the Continuous Dependence of the Solution on the Data
}

\author{
Junichi Aramaki \\ Division of Science, Faculty of Science and Engineering, Tokyo Denki University \\ Hatoyama-machi, Saitama 350-0394, Japan.
}

\begin{abstract}
In this paper, we prove the existence of a solution for a variational inequality associated with the Maxwell-Stokes type equation in a bounded multiply connected domain with holes. Our equation is nonlinear and contains, the so called, $p$-curlcurl equation. Furthermore, we obtain the continuous dependence of the solution on the data.
\end{abstract}

2010 Mathematics Subject Classification: 35A05, 35A15, 35H30, 35E10, $35 \mathrm{~J} 25$

Keywords: Variational inequality, Maxwell-Stokes type equation, penalty method, minimization problem.

\section{INTRODUCTION}

In this paper, we consider a stationary nonlinear electromagnetic field in a multiply connected domain in $\mathbb{R}^{3}$ with holes. The electric and magnetic fields $\boldsymbol{e}$ and $\boldsymbol{h}$ satisfy the following Maxwell equations

$$
\begin{cases}\boldsymbol{j}=\operatorname{curl} \boldsymbol{h} & \text { in } \Omega, \\ \operatorname{div} \boldsymbol{h}=0 & \text { in } \Omega, \\ \operatorname{div} \boldsymbol{e}=q & \text { in } \Omega, \\ \operatorname{curl} \boldsymbol{e}=\boldsymbol{f} & \text { in } \Omega,\end{cases}
$$


where $\boldsymbol{j}$ denotes the total current density, $q$ is the electric charge and $\boldsymbol{f}$ is here a given external field. We use the following nonlinear extension of Ohm's law

$$
|\boldsymbol{j}|^{p-2} \boldsymbol{j}=\sigma \boldsymbol{e}
$$

where $\sigma$ is the electric conductivity. Then the magnetic field $\boldsymbol{h}$ satisfies

$$
\begin{cases}\operatorname{curl}\left[\frac{1}{\sigma}|\operatorname{curl} \boldsymbol{h}|^{p-2} \operatorname{curl} \boldsymbol{h}\right]=\boldsymbol{f} & \text { in } \Omega, \\ \operatorname{div} \boldsymbol{h}=0 & \text { in } \Omega .\end{cases}
$$

The left-hand side of the first equation in (1.1) is called the $p$-curlcurl operator. For a weak solution to such a system under certain boundary condition, see Yin et al. [17], Miranda et al. [10], [11], Pan [13], and Aramaki [4]. A necessary condition for the existence of a solution of the problem (1.1) is that the external field $f$ must satisfy $\operatorname{div} \boldsymbol{f}=0$ in $\Omega$. However, if this condition is not satisfied, then it is expected to demand an unknown potential function $\pi$ such that

$$
\begin{cases}\operatorname{curl}\left[\frac{1}{\sigma}|\operatorname{curl} \boldsymbol{h}|^{p-2} \operatorname{curl} \boldsymbol{h}\right]+\nabla \pi=\boldsymbol{f} & \text { in } \Omega \\ \operatorname{div} \boldsymbol{h}=0 & \text { in } \Omega .\end{cases}
$$

Whether a solution to (1.2) exists or not depends heavily on the boundary conditions and the geometry of the domain $\Omega$.

We also consider another constitutive law that arises in type-II superconductors, which is known as an extension of the Bean critical-state model in Prigozhin [14]. In this case the current density $\boldsymbol{j}=\operatorname{curl} \boldsymbol{h}$ cannot exceed the critical value $\Psi=\Psi(x)>0$ and we have

$$
\boldsymbol{e}= \begin{cases}\frac{1}{\sigma}|\operatorname{curl} \boldsymbol{h}|^{p-2} \operatorname{curl} \boldsymbol{h} & \text { if }|\operatorname{curl} \boldsymbol{h}|<\Psi(x), \\ \left(\frac{1}{\sigma} \Psi^{p-2}+\lambda\right) \operatorname{curl} \boldsymbol{h} & \text { if }|\operatorname{curl} \boldsymbol{h}|=\Psi(x),\end{cases}
$$

where $\lambda=\lambda(x) \geq 0$ is regarded as a unknown Lagrange multiplier. This leads to the variational inequality

$$
\int_{\Omega} \frac{1}{\sigma}|\operatorname{curl} \boldsymbol{h}|^{p-2} \operatorname{curl} \boldsymbol{h} \cdot \operatorname{curl}(\boldsymbol{v}-\boldsymbol{h}) d x+\int_{\Omega} \boldsymbol{\nabla} \pi \cdot(\boldsymbol{v}-\boldsymbol{h}) d x \geq \int_{\Omega} \boldsymbol{f} \cdot(\boldsymbol{v}-\boldsymbol{h}) d x
$$

for any test function $\boldsymbol{v}$ such that $|\operatorname{curl} \boldsymbol{v}| \leq \Psi(x)$ a.e. in $\Omega$.

In this paper, we consider such a variational inequality. We use a nicely extended Carathéodory function $S(x, t)$ defined in $\Omega \times[0, \infty)$ by Aramaki [5], and we consider the following system

$$
\begin{cases}\operatorname{curl}\left[S_{t}\left(x,|\operatorname{curl} \boldsymbol{u}|^{2}\right) \operatorname{curl} \boldsymbol{u}\right]+\nabla \pi=\boldsymbol{f} & \text { in } \Omega, \\ \operatorname{div} \boldsymbol{u}=0 & \text { in } \Omega\end{cases}
$$


where $S_{t}=\partial S / \partial t$. Since we allow that $\Omega$ is multiply connected and has holes, we assume that $\Omega$ satisfies (O1) and (O2) defined in section 2. In particular, the boundary $\Gamma$ of $\Omega$ has finitely many connected components $\Gamma_{0}, \Gamma_{1}, \ldots, \Gamma_{I}$ with $\Gamma_{0}$ denoting the boundary of the infinite connected component of $\mathbb{R}^{3} \backslash \bar{\Omega}$.

We impose boundary conditions to system (1.3),

$$
\begin{cases}\boldsymbol{u} \times \boldsymbol{n}=\mathbf{0} & \text { on } \Gamma, \\ \langle\boldsymbol{u} \cdot \boldsymbol{n}, 1\rangle_{\Gamma_{i}}=0 & \text { for } i=1, \ldots, I,\end{cases}
$$

where $\boldsymbol{n}$ is the outer unit normal vector to $\Gamma$ and $\langle\cdot, \cdot\rangle_{\Gamma_{i}}$ denotes some duality bracket defined in section 2 .

Thus we consider the following variational inequality: to find $(\boldsymbol{u}, \pi)$ in an appropriate space such that

$$
\begin{aligned}
\int_{\Omega} S_{t}\left(x,|\operatorname{curl} \boldsymbol{u}|^{2}\right) \operatorname{curl} \boldsymbol{u} \cdot \operatorname{curl}(\boldsymbol{v}-\boldsymbol{u}) d x-\int_{\Omega} \pi \operatorname{div}(\boldsymbol{v} & -\boldsymbol{u}) d x \\
& \quad \geq \int_{\Omega} \boldsymbol{f} \cdot(\boldsymbol{v}-\boldsymbol{u}) d x
\end{aligned}
$$

for all $\boldsymbol{v}$ such that $|\operatorname{curl} \boldsymbol{v}| \leq \Psi(x)$ a.e. in $\Omega$.

The first purpose of this paper is to show the existence of a unique solution to (1.5) under boundary conditions (1.4) (Theorem 3 3). More precisely, let the constrained function $\Psi$ be of the form $\Psi(x)=F(\varphi(x))$, where $F: \mathbb{R} \rightarrow[0, \infty)$ is a continuous function and $\varphi \in L^{\infty}(\Omega)$, To get a solution to (1.5), we use the standard minimization problem of some functional on a closed convex subset

$$
\mathbb{K}_{\varphi}=\left\{\boldsymbol{v} \in \mathbb{X}_{N}^{p}(\Omega):|\operatorname{curl} \boldsymbol{v}| \leq F(\varphi) \text { a.e. in } \Omega\right\},
$$

where $\mathbb{X}_{N}^{p}(\Omega)$ is a reflexive Banach space associated with the boundary condition (1.4) defined in section 2 . We note that the functional

$$
\int_{\Omega} S\left(x,|\operatorname{curl} \boldsymbol{v}|^{2}\right) d x
$$

is not coercive on $\mathbb{K}_{\varphi}$. To overcome this, we use the penalty method introduced by Temam [16]. As a result, we can find a unique solution to (1.5) as $(\boldsymbol{u}, \pi) \in \widehat{\mathbb{K}}_{\varphi} \times L^{p^{\prime}}(\Omega)$, where

$$
\widehat{\mathbb{K}}_{\varphi}=\left\{\boldsymbol{v} \in \mathbb{V}_{N}^{p}(\Omega):|\operatorname{curl} \boldsymbol{v}| \leq F(\varphi) \text { a.e. in } \Omega\right\} .
$$

Here $\mathbb{V}_{N}^{p}(\Omega)$ is a reflexive Banach space defined in section 2.

The second purpose of this paper is to derive the continuity of the solution to (1.5) on the data $\boldsymbol{f}$ and $\varphi$. Let $\boldsymbol{f}_{n}, \boldsymbol{f} \in \mathbb{X}_{N}^{p}(\Omega)^{\prime}\left(\mathbb{X}_{N}^{p}(\Omega)^{\prime}\right.$ denotes the dual space of $\mathbb{X}_{N}^{p}(\Omega)$ ), and 
let $\left(\boldsymbol{u}_{n}, \pi_{n}\right) \in \widehat{\mathbb{K}}_{\varphi_{n}} \times L^{p^{\prime}}(\Omega)$ be the solution to (1.5) with $\boldsymbol{f}=\boldsymbol{f}_{n}$ and $\varphi=\varphi_{n}$. We show that if $\boldsymbol{f}_{n} \rightarrow \boldsymbol{f}$ in $\mathbb{X}_{N}^{p}(\Omega)^{\prime}$ and $\varphi_{n} \rightarrow \varphi$ in $L^{\infty}(\Omega)$, then we can prove that $\boldsymbol{u}_{n} \rightarrow \boldsymbol{u}$ strongly in $\mathbb{V}_{N}^{p}(\Omega)$ and $\pi_{n} \rightarrow \pi$ weakly in $L^{p^{\prime}}(\Omega)$. To show that $\left\{\boldsymbol{u}_{n}\right\}$ converges strongly in $\mathbb{V}_{N}^{p}(\Omega)$, we use the celebrated result of Mosco [12] (Theorem 4.2).

This paper is organized as follows. Section 2 covers preliminaries in which we give the geometry of the domain $\Omega$, some spaces of functions and their properties. In section 3 , we consider a variational inequality as in (1.5) and give the main theorem on the existence of a solution. In section 4 , we consider the continuity of the solution obtained in section 3 on the data $f$ and the constrained function. We apply the result of Mosco [12].

\section{PRELIMINARIES}

In this section, we introduce the geometry of the domain, a Carathéodory function $S(x, t)$ on $\Omega \times[0,+\infty)$ satisfying some structural conditions, and some spaces of functions.

Let $\Omega$ be a bounded domain in $\mathbb{R}^{3}$ with a $C^{1,1}$ boundary $\Gamma$. Since we allow $\Omega$ to be a multiply-connected domain with holes in $\mathbb{R}^{3}$, we assume that $\Omega$ satisfies the following conditions as in Amrouche and Seloula [2] (cf. Amrouche and Seloula [1], Dautray and Lions [7, vol. 3] and Girault and Raviart [9]). $\Omega$ is locally situated on one side of $\Gamma$ and satisfies the following $(\mathrm{O} 1)$ and $(\mathrm{O} 2)$.

(O1) $\Gamma$ has a finite number of connected components $\Gamma_{0}, \Gamma_{1}, \ldots, \Gamma_{I}$ with $\Gamma_{0}$ denoting the boundary of the infinite connected component of $\mathbb{R}^{3} \backslash \bar{\Omega}$.

(O2) There exist $J$ connected open surfaces $\Sigma_{j},(j=1, \ldots, J)$, called cuts, contained in $\Omega$ such that

(a) each surface $\Sigma_{j}$ is an open subset of a smooth manifold $\mathcal{M}_{j}$,

(b) $\partial \Sigma_{j} \subset \Gamma(j=1, \ldots, J)$, where $\partial \Sigma_{j}$ denotes the boundary of $\Sigma_{j}$, and $\Sigma_{j}$ is non-tangential to $\Gamma$,

(c) $\overline{\Sigma_{j}} \cap \overline{\Sigma_{k}}=\emptyset(j \neq k)$,

(d) the open set $\Omega^{\circ}=\Omega \backslash\left(\cup_{j=1}^{J} \Sigma_{j}\right)$ is simply connected and has Lipschitz-continuous boundary.

The number $J$ is called the first Betti number and $I$ is the second Betti number. We say 
that $\Omega$ is simply connected if $J=0$ and $\Omega$ has no holes if $I=0$. If we define

$$
\mathbb{K}_{T}^{p}(\Omega)=\left\{\boldsymbol{v} \in \boldsymbol{W}^{1, p}(\Omega) ; \operatorname{curl} \boldsymbol{v}=\mathbf{0}, \operatorname{div} \boldsymbol{v}=0 \text { in } \Omega, \boldsymbol{v} \cdot \boldsymbol{n}=0 \text { on } \Gamma\right\}
$$

and

$$
\mathbb{K}_{N}^{p}(\Omega)=\left\{\boldsymbol{v} \in \boldsymbol{W}^{1, p}(\Omega) ; \operatorname{curl} \boldsymbol{v}=\mathbf{0}, \operatorname{div} \boldsymbol{v}=0 \text { in } \Omega, \boldsymbol{v} \times \boldsymbol{n}=\mathbf{0} \text { on } \Gamma\right\}
$$

then it is well known that $\operatorname{dim} \mathbb{K}_{T}^{p}(\Omega)=J$ and $\operatorname{dim} \mathbb{K}_{N}^{p}(\Omega)=I$.

Throughout this paper, let $1<p<\infty$ and we denote the conjugate exponent of $p$ by $p^{\prime}$, i.e., $(1 / p)+\left(1 / p^{\prime}\right)=1$. From now on we use $L^{p}(\Omega), W_{0}^{1, p}(\Omega)$ and $W^{1, p}(\Omega)$ for the standard $L^{p}$ and Sobolev spaces of functions. For any Banach space $B$, we denote $B \times B \times B$ by the boldface character $\boldsymbol{B}$. We use this character to denote vector and vector-valued functions, and we denote the standard Euclidean inner product of vectors $\boldsymbol{a}$ and $\boldsymbol{b}$ in $\mathbb{R}^{3}$ by $\boldsymbol{a} \cdot \boldsymbol{b}$. For the dual space $\boldsymbol{B}^{\prime}$ of $\boldsymbol{B}$, we write $\langle\cdot, \cdot\rangle_{\boldsymbol{B}^{\prime}, \boldsymbol{B}}$ for the duality bracket.

We assume that a Carathéodory function $S(x, t)$ in $\Omega \times[0, \infty)$ satisfies the following structural conditions. For a.e. $x \in \Omega, S(x, t) \in C^{2}((0, \infty)) \cap C^{0}([0, \infty))$, and positive constants $0<\lambda \leq \Lambda<\infty$ such that for a.e. $x \in \Omega$,

$$
\begin{array}{r}
S(x, 0)=0 \text { and } \quad \lambda t^{(p-2) / 2} \leq S_{t}(x, t) \leq \Lambda t^{(p-2) / 2} \text { for } t>0, \\
\lambda t^{(p-2) / 2} \leq S_{t}(x, t)+2 t S_{t t}(x, t) \leq \Lambda t^{(p-2) / 2} \text { for } t>0, \\
\text { If } 1<p<2, S_{t t}(x, t)<0, \text { and if } p \geq 2, S_{t t}(x, t) \geq 0 \text { for } t>0,
\end{array}
$$

where $S_{t}=\partial S / \partial t$ and $S_{t t}=\partial^{2} S / \partial t^{2}$. We note that from (2.1a), it follows that

$$
\frac{2}{p} \lambda t^{p / 2} \leq S(x, t) \leq \frac{2}{p} \Lambda t^{p / 2} \text { for } t \geq 0 .
$$

Example 2.1. If $S(x, t)=\nu(x) g(t) t^{p / 2}$, where $\nu$ is a measurable function in $\Omega$ and satisfies $0<\nu_{*} \leq \nu(x) \leq \nu^{*}<\infty$ for a.e. $x \in \Omega$ for some constants $\nu_{*}$ and $\nu^{*}$, and $g \in C^{\infty}([0, \infty))$,

When $g(t) \equiv 1$, it follows from elementary calculations that (2.1a)-(2.1c) hold.

As another example, we can take

$$
g(t)= \begin{cases}a\left(e^{-1 / t}+1\right) & \text { if } t>0 \\ a & \text { if } t=0\end{cases}
$$

with a constant $a>0$. Then $S(x, t)=\nu(x) g(t) t^{p / 2}$ satisfies (2.1a)-(2.1c) if $p \geq 2$. (cf. Aramaki [6, Example 3.2]). 
We remember the monotonic property of $S_{t}$.

Lemma 2.2. There exists a constant $c>0$ such that for all $\boldsymbol{a}, \boldsymbol{b} \in \mathbb{R}^{3}$,

$$
\begin{aligned}
& \left(S_{t}\left(x,|\boldsymbol{a}|^{2}\right) \boldsymbol{a}-S_{t}\left(x,|\boldsymbol{b}|^{2}\right) \boldsymbol{b}\right) \cdot(\boldsymbol{a}-\boldsymbol{b}) \\
& \geq \geq \begin{cases}c|\boldsymbol{a}-\boldsymbol{b}|^{p} & \text { if } p \geq 2, \\
c(|\boldsymbol{a}|+|\boldsymbol{b}|)^{p-2}|\boldsymbol{a}-\boldsymbol{b}|^{2} & \text { if } 1<p<2 .\end{cases}
\end{aligned}
$$

In particular, if $\boldsymbol{a} \neq \boldsymbol{b}$, we have

$$
\left(S_{t}\left(x,|\boldsymbol{a}|^{2}\right) \boldsymbol{a}-S_{t}\left(x,|\boldsymbol{b}|^{2}\right) \boldsymbol{b}\right) \cdot(\boldsymbol{a}-\boldsymbol{b})>0 .
$$

For the proof, see Aramaki [5, Lemma 3.6].

Lemma 2.3. There exists a constant $C_{1}>0$ depending only on $\Lambda$ and $p$ such that for any $\boldsymbol{a}, \boldsymbol{b} \in \mathbb{R}^{3}$,

$$
\left|S_{t}\left(x,|\boldsymbol{a}|^{2}\right) \boldsymbol{a}-S_{t}\left(x,|\boldsymbol{b}|^{2}\right) \boldsymbol{b}\right| \leq \begin{cases}C_{1}|\boldsymbol{a}-\boldsymbol{b}|^{p-1} & \text { if } 1<p<2 \\ C_{1}(|\boldsymbol{a}|+|\boldsymbol{b}|)^{p-2}|\boldsymbol{a}-\boldsymbol{b}| & \text { if } p \geq 2\end{cases}
$$

For the proof, see Aramaki [3].

We can see the convexity of $S(x, t)$ in the following sense.

Lemma 2.4. If $S(x, t)$ satisfies (2.1a) and (2.1b), then for a.e. $x \in \Omega$, the function $\mathbb{R} \ni t \mapsto g[t]=S\left(x, t^{2}\right)$ is strictly convex.

For the proof, see [6, Lemma 2.3].

The following inequality is used frequently (cf. [2]). If $\Omega$ is a bounded domain in $\mathbb{R}^{3}$ with a $C^{1,1}$ boundary $\Gamma$, and if $\boldsymbol{u} \in \boldsymbol{L}^{p}(\Omega)$ satisfies curl $\boldsymbol{u} \in \boldsymbol{L}^{p}(\Omega), \operatorname{div} \boldsymbol{u} \in L^{p}(\Omega)$ and $\boldsymbol{u} \times \boldsymbol{n} \in \boldsymbol{W}^{1-1 / p, p}(\Gamma)$, then $\boldsymbol{u} \in \boldsymbol{W}^{1, p}(\Omega)$ and there exists a constant $C>0$ depending only on $p$ and $\Omega$ such that

$$
\begin{aligned}
&\|\boldsymbol{u}\|_{\boldsymbol{W}^{1, p}(\Omega)} \leq C\left(\|\operatorname{curl} \boldsymbol{u}\|_{\boldsymbol{L}^{p}(\Omega)}+\|\operatorname{div} \boldsymbol{u}\|_{L^{p}(\Omega)}+\|\boldsymbol{u}\|_{\boldsymbol{L}^{p}(\Omega)}\right. \\
&\left.+\|\boldsymbol{u} \times \boldsymbol{n}\|_{W^{1-1 / p, p}(\Gamma)}\right) .
\end{aligned}
$$

Moreover, if $\boldsymbol{u} \in \boldsymbol{L}^{p}(\Omega)$ satisfies curl $\boldsymbol{u} \in \boldsymbol{L}^{p}(\Omega)$, then $\boldsymbol{u} \times \boldsymbol{n} \in \boldsymbol{W}^{-1 / p, p}(\Gamma)$ is well defined, and if $\boldsymbol{u} \in \boldsymbol{L}^{p}(\Omega)$ satisfies $\operatorname{div} \boldsymbol{u} \in L^{p}(\Omega)$, then $\boldsymbol{u} \cdot \boldsymbol{n} \in W^{-1 / p, p}(\Gamma)$ is well defined by the formulae

$$
\langle\boldsymbol{u} \times \boldsymbol{n}, \boldsymbol{\phi}\rangle_{\boldsymbol{W}^{-1 / p, p}(\Gamma), \boldsymbol{W}^{1-1 / p^{\prime}, p^{\prime}(\Gamma)}}=\int_{\Omega} \boldsymbol{u} \cdot \operatorname{curl} \boldsymbol{\phi} d x-\int_{\Omega} \operatorname{curl} \boldsymbol{u} \cdot \boldsymbol{\phi} d x
$$


for all $\boldsymbol{\phi} \in \boldsymbol{W}^{1, p^{\prime}}(\Omega)$ and

$$
\langle\boldsymbol{u} \cdot \boldsymbol{n}, \phi\rangle_{W^{-1 / p, p}(\Gamma), W^{1-1 / p^{\prime}, p^{\prime}(\Gamma)}}=\int_{\Omega} \boldsymbol{u} \cdot \boldsymbol{\nabla} \phi d x+\int_{\Omega}(\operatorname{div} \boldsymbol{u}) \phi d x
$$

for all $\phi \in W^{1, p^{\prime}}(\Omega)$. Furthermore, if $\boldsymbol{u} \in \boldsymbol{W}^{1, p}(\Omega)$ satisfies $\boldsymbol{u} \times \boldsymbol{n}=\mathbf{0}$ on $\Gamma$, then there exists a constant $C>0$ depending only on $p$ and $\Omega$ such that

$$
\|\boldsymbol{u}\|_{\boldsymbol{L}^{p}(\Omega)} \leq C\left(\|\operatorname{curl} \boldsymbol{u}\|_{\boldsymbol{L}^{p}(\Omega)}+\|\operatorname{div} \boldsymbol{u}\|_{L^{p}(\Omega)}+\sum_{i=1}^{I}\left|\langle\boldsymbol{u} \cdot \boldsymbol{n}, 1\rangle_{\Gamma_{i}}\right|\right.
$$

where $\langle\cdot, \cdot\rangle_{\Gamma_{i}}=\langle\cdot, \cdot\rangle_{W^{-1 / p, p}\left(\Gamma_{i}\right), W^{1-1 / p^{\prime}, p^{\prime}}\left(\Gamma_{i}\right)} \cdot$

Define a space

$$
\begin{aligned}
\mathbb{X}_{N}^{p}(\Omega)=\left\{\boldsymbol{v} \in \boldsymbol{L}^{p}(\Omega) ; \operatorname{curl} \boldsymbol{u} \in \boldsymbol{L}^{p}(\Omega), \operatorname{div} \boldsymbol{v} \in L^{p}(\Omega), \boldsymbol{u} \times \boldsymbol{n}=\mathbf{0} \text { on } \Gamma,\right. \\
\left.\langle\boldsymbol{u} \cdot \boldsymbol{n}, 1\rangle_{\Gamma_{i}}=0 \text { for } i=1, \ldots, I\right\} .
\end{aligned}
$$

with the norm

$$
\|\boldsymbol{v}\|_{\mathbb{X}_{N}^{p}(\Omega)}=\left(\|\operatorname{curl} \boldsymbol{u}\|_{L^{p}(\Omega)}^{p}+\|\operatorname{div} \boldsymbol{u}\|_{L^{p}(\Omega)}^{p}\right)^{1 / p} .
$$

We note that $\|\boldsymbol{v}\|_{\mathbb{X}_{N}^{p}(\Omega)}$ is equivalent to $\|\boldsymbol{v}\|_{W^{1, p}(\Omega)}$ for $\boldsymbol{v} \in \mathbb{X}_{N}^{p}(\Omega)$ (cf. [2]). Since $\mathbb{X}_{N}^{p}(\Omega)$ is a closed subspace of $\boldsymbol{W}^{1, p}(\Omega)$, we can see that $\mathbb{X}_{N}^{p}(\Omega)$ is a reflexive Banach space and $\boldsymbol{W}_{0}^{1, p}(\Omega) \hookrightarrow \mathbb{X}_{N}^{p}(\Omega) \hookrightarrow \boldsymbol{W}^{1, p}(\Omega)$, where the symbol $\hookrightarrow$ means that the inclusion map is continuous. Furthermore, we define a closed subspace $\mathbb{V}_{N}^{p}(\Omega)$ of $\mathbb{X}_{N}^{p}(\Omega)$ by

$$
\mathbb{V}_{N}^{p}(\Omega)=\left\{\boldsymbol{v} \in \mathbb{X}_{N}^{p}(\Omega) ; \operatorname{div} \boldsymbol{v}=0 \text { in } \Omega\right\}
$$

with the norm $\|\boldsymbol{v}\|_{\mathbb{V}_{N}^{p}(\Omega)}=\|\operatorname{curl} \boldsymbol{v}\|_{\boldsymbol{L}^{p}(\Omega)}$ which is also equivalent to $\|\boldsymbol{v}\|_{\boldsymbol{W}^{1, p}(\Omega)}$. We note that $\mathbb{V}_{N}^{p}(\Omega)$ is also a reflexive Banach space.

Lemma 2.5. If $\boldsymbol{v} \in \boldsymbol{L}^{p^{\prime}}(\Omega)$, then $\operatorname{curl} \boldsymbol{v} \in \mathbb{X}_{N}^{p}(\Omega)^{\prime}$ and

$$
\langle\operatorname{curl} \boldsymbol{v}, \boldsymbol{\varphi}\rangle_{\mathbb{X}_{N}^{p}(\Omega)^{\prime}, \mathbb{X}_{N}^{p}(\Omega)}=\int_{\Omega} \boldsymbol{v} \cdot \operatorname{curl} \boldsymbol{\varphi} d x \text { for all } \boldsymbol{\varphi} \in \mathbb{X}_{N}^{p}(\Omega)
$$

Moreover, there exists a constant $C>0$ depending only on $p$ and $\Omega$ such that

$$
\|\operatorname{curl} \boldsymbol{v}\|_{\mathbb{X}_{N}^{p}(\Omega)^{\prime}} \leq C\|\boldsymbol{v}\|_{\boldsymbol{L}^{p^{\prime}(\Omega)}} \text { for all } \boldsymbol{v} \in \boldsymbol{L}^{p^{\prime}}(\Omega) .
$$

Proof. Let $\boldsymbol{v} \in \boldsymbol{L}^{p^{\prime}}(\Omega)$. Then the distribution curl $\boldsymbol{v} \in \mathcal{D}^{\prime}(\Omega)$ is defined by

$$
\langle\operatorname{curl} \boldsymbol{v}, \boldsymbol{\varphi}\rangle=\int_{\Omega} \boldsymbol{v} \cdot \operatorname{curl} \boldsymbol{\varphi} d x \text { for all } \boldsymbol{\varphi} \in \mathcal{D}(\Omega)=\boldsymbol{C}_{0}^{\infty}(\Omega) .
$$


Define temporarily a Banach space

$$
H_{0}(\operatorname{curl}, \Omega)=\left\{\boldsymbol{v} \in \boldsymbol{L}^{p}(\Omega) ; \operatorname{curl} \boldsymbol{v} \in \boldsymbol{L}^{p}(\Omega), \boldsymbol{v} \times \boldsymbol{n}=\mathbf{0} \text { on } \Gamma\right\}
$$

with the norm $\|\boldsymbol{v}\|_{H_{0}(\operatorname{curl}, \Omega)}=\left(\|\boldsymbol{v}\|_{\boldsymbol{L}^{p}(\Omega)}^{2}+\|\operatorname{curl} \boldsymbol{v}\|_{\boldsymbol{L}^{p}(\Omega)}^{p}\right)^{1 / p}$. Then by Temam [16] or [9], $\mathcal{D}(\Omega)$ is dense in $H_{0}(\operatorname{curl}, \Omega)$. Hence for any $\varphi \in H_{0}(\operatorname{curl}, \Omega)$, there exists a sequence $\left\{\boldsymbol{\varphi}_{j}\right\} \subset \mathcal{D}(\Omega)$ such that $\boldsymbol{\varphi}_{j} \rightarrow \boldsymbol{\varphi}$ in $H_{0}(\operatorname{curl}, \Omega)$. Define

$$
\langle\operatorname{curl} \boldsymbol{v}, \boldsymbol{\varphi}\rangle=\lim _{j \rightarrow \infty} \int_{\Omega} \boldsymbol{v} \cdot \operatorname{curl} \boldsymbol{\varphi}_{j} d x .
$$

Clearly, the definition is well defined (independent of the choice of a sequence $\left\{\boldsymbol{\varphi}_{j}\right\}$ such that $\varphi_{j} \rightarrow \varphi$ in $H_{0}(\operatorname{curl}, \Omega)$ ), and

$$
\begin{aligned}
|\langle\operatorname{curl} \boldsymbol{v}, \boldsymbol{\varphi}\rangle|=\lim _{j \rightarrow \infty}\left|\int_{\Omega} \boldsymbol{v} \cdot \operatorname{curl} \boldsymbol{\varphi}_{j} d x\right| \leq \lim _{j \rightarrow \infty}\|\boldsymbol{v}\|_{\boldsymbol{L}^{p^{\prime}(\Omega)}}\left\|\operatorname{curl} \boldsymbol{\varphi}_{j}\right\|_{\boldsymbol{L}^{p}(\Omega)} \\
\leq\|\boldsymbol{v}\|_{\boldsymbol{L}^{p^{\prime}}(\Omega)}\|\operatorname{curl} \boldsymbol{\varphi}\|_{\boldsymbol{L}^{p}(\Omega)} .
\end{aligned}
$$

Therefore, we have

$$
\langle\operatorname{curl} \boldsymbol{v}, \boldsymbol{\varphi}\rangle=\lim _{j \rightarrow \infty} \int_{\Omega} \boldsymbol{v} \cdot \operatorname{curl} \boldsymbol{\varphi}_{j} d x=\int_{\Omega} \boldsymbol{v} \cdot \operatorname{curl} \boldsymbol{\varphi} d x \text { for all } \boldsymbol{\varphi} \in H_{0}(\operatorname{curl}, \Omega) .
$$

Moreover, we have $|\langle\operatorname{curl} \boldsymbol{v}, \boldsymbol{\varphi}\rangle| \leq\|\boldsymbol{v}\|_{\boldsymbol{L}^{p^{\prime}(\Omega)}}\|\boldsymbol{\varphi}\|_{H_{0}(\operatorname{curl}, \Omega)}$. Thus we can see that curl $\boldsymbol{v} \in H_{0}(\operatorname{curl}, \Omega)^{\prime}$. On the other hand, since $\mathbb{X}_{N}^{p}(\Omega) \hookrightarrow H_{0}(\operatorname{curl}, \Omega)$, we have $H_{0}(\operatorname{curl}, \Omega)^{\prime} \hookrightarrow \mathbb{X}_{N}^{p}(\Omega)^{\prime}$, and there exists a constant $C>0$ depending only on $p$ and $\Omega$ such that

$$
|\langle\operatorname{curl} \boldsymbol{v}, \boldsymbol{\varphi}\rangle| \leq C\|\boldsymbol{v}\|_{\boldsymbol{L}^{p^{\prime}(\Omega)}}\|\boldsymbol{\varphi}\|_{\mathbb{X}_{N}^{p}(\Omega)} \text { for all } \boldsymbol{\varphi} \in \mathbb{X}_{N}^{p}(\Omega) .
$$

Thus curl $\boldsymbol{v} \in \mathbb{X}_{N}^{p}(\Omega)^{\prime}$, (2.4) holds and $\|\operatorname{curl} \boldsymbol{v}\|_{\mathbb{X}_{N}^{p}(\Omega)^{\prime}} \leq C\|\boldsymbol{v}\|_{\boldsymbol{L}^{p^{\prime}}(\Omega)}$ for any $\boldsymbol{v} \in$ $\boldsymbol{L}^{p^{\prime}}(\Omega)$.

Corollary 2.6. If $\boldsymbol{v} \in \mathbb{X}_{N}^{p}(\Omega)$, then $\operatorname{curl}\left[S_{t}\left(x,|\operatorname{curl} \boldsymbol{v}|^{2}\right) \operatorname{curl} \boldsymbol{v}\right] \in \mathbb{X}_{N}^{p}(\Omega)^{\prime}$, and there exists a constant $C>0$ depending only on $p, \Lambda$ and $\Omega$ such that

$$
\left\|\operatorname{curl}\left[S_{t}\left(x|\operatorname{curl} \boldsymbol{v}|^{2}\right) \operatorname{curl} \boldsymbol{v}\right]\right\|_{\mathbb{X}_{N}^{p}(\Omega)^{\prime}} \leq C\|\boldsymbol{v}\|_{\mathbb{X}_{N}^{p}(\Omega)}^{p-1} .
$$

Proof. If $\boldsymbol{v} \in \mathbb{X}_{N}^{p}(\Omega)$, then from (2.1b), $\left|S_{t}\left(x,|\operatorname{curl} \boldsymbol{v}|^{2}\right) \operatorname{curl} \boldsymbol{v}\right| \leq \Lambda|\operatorname{curl} \boldsymbol{v}|^{p-1}$. Hence $S_{t}\left(x,|\operatorname{curl} \boldsymbol{v}|^{2}\right) \operatorname{curl} \boldsymbol{v} \in \boldsymbol{L}^{p^{\prime}}(\Omega)$, and

$$
\left\|S_{t}\left(x,|\operatorname{curl} \boldsymbol{v}|^{2}\right) \operatorname{curl} \boldsymbol{v}\right\|_{\boldsymbol{L}^{p^{\prime}(\Omega)}} \leq \Lambda\left(\int_{\Omega}|\operatorname{curl} \boldsymbol{v}|^{p} d x\right)^{1 / p^{\prime}} \leq \Lambda\|\boldsymbol{v}\|_{\mathbb{X}_{N}^{p}(\Omega)}^{p-1} .
$$

It suffices to apply Lemma 2.5 . 


\section{A VARIATIONAL INEQUALITY FOR THE MAXWELL-STOKES PROBLEM}

In this section, we consider a variational inequality. Let $F: \mathbb{R} \rightarrow[0, \infty)$ be a continuous function, and let $\varphi \in L^{\infty}(\Omega)$. Define a closed convex subset $\mathbb{K}_{\varphi}$ of $\mathbb{X}_{N}^{p}(\Omega)$ and a closed convex subset $\widehat{\mathbb{K}}_{\varphi}$ of $\mathbb{V}_{N}^{p}(\Omega)$ by

$$
\mathbb{K}_{\varphi}=\left\{\boldsymbol{v} \in \mathbb{X}_{N}^{p}(\Omega) ;|\operatorname{curl} \boldsymbol{v}| \leq F(\varphi) \text { a.e. in } \Omega\right\}
$$

and

$$
\widehat{\mathbb{K}}_{\varphi}=\left\{\boldsymbol{v} \in \mathbb{V}_{N}^{p}(\Omega) ;|\operatorname{curl} \boldsymbol{v}| \leq F(\varphi) \text { a.e. in } \Omega\right\},
$$

respectively. For a given function $\boldsymbol{f} \in \mathbb{X}_{N}^{p}(\Omega)^{\prime}$, we consider the following variational inequality: to find $(\boldsymbol{u}, \pi) \in \widehat{\mathbb{K}}_{\varphi} \times L^{p^{\prime}}(\Omega)$ such that

$$
\begin{aligned}
\int_{\Omega} S_{t}\left(x,|\operatorname{curl} \boldsymbol{u}|^{2}\right) \operatorname{curl} \boldsymbol{u} \cdot \operatorname{curl}(\boldsymbol{v}- & \boldsymbol{u}) d x-\int_{\Omega} \pi \operatorname{div} \boldsymbol{v} d x \\
& \geq\langle\boldsymbol{f}, \boldsymbol{v}-\boldsymbol{u}\rangle_{\mathbb{X}_{N}^{p}(\Omega)^{\prime}, \mathbb{X}_{N}^{p}(\Omega)} \text { for all } \boldsymbol{v} \in \mathbb{K}_{\varphi} .
\end{aligned}
$$

We solve problem (3.1) by the penalty method introduced by Temam [16]. To do so, we consider the following functional $E_{\varepsilon}$ on $\mathbb{K}_{\varphi}$ depending on a parameter $\varepsilon \in(0,1]$ defined by

$$
\begin{aligned}
E_{\varepsilon}[\boldsymbol{v}]=\frac{1}{2}\left\{\int_{\Omega} S\left(x,|\operatorname{curl} \boldsymbol{v}|^{2}\right) d x+\frac{1}{\varepsilon} \int_{\Omega} S(\right. & \left.\left.x,(\operatorname{div} \boldsymbol{v})^{2}\right) d x\right\} \\
& -\langle\boldsymbol{f}, \boldsymbol{v}\rangle_{\mathbb{X}_{N}^{p}(\Omega)^{\prime}, \mathbb{X}_{N}^{p}(\Omega)} \text { for } \boldsymbol{v} \in \mathbb{K}_{\varphi} .
\end{aligned}
$$

We derive the following minimization problem: to find $\boldsymbol{u}_{\varepsilon} \in \mathbb{K}_{\varphi}$ such that

$$
E_{\varepsilon}\left[\boldsymbol{u}_{\varepsilon}\right]=\inf _{\boldsymbol{v} \in \mathbb{K}_{\varphi}} E_{\varepsilon}[\boldsymbol{v}]
$$

We call such a $\boldsymbol{u}_{\varepsilon} \in \mathbb{K}_{\varphi}$ a minimizer of $E_{\varepsilon}$.

Proposition 3.1. Assume that $\boldsymbol{f} \in \mathbb{X}_{N}^{p}(\Omega)^{\prime}$. Then the minimization problem (3.3) has a unique minimizer $\boldsymbol{u}_{\varepsilon} \in \mathbb{K}_{\varphi}$, and there exists a constant $C>0$ depending only on $p, \lambda$ and $\Omega$, but independent of $\varepsilon \in(0,1]$ such that

$$
\left\|\boldsymbol{u}_{\varepsilon}\right\|_{\mathbb{X}_{N}^{p}(\Omega)}^{p} \leq C\|\boldsymbol{f}\|_{\mathbb{X}_{N}^{p}(\Omega)^{\prime}}^{p^{\prime}}
$$

and

$$
\left\|\operatorname{div} \boldsymbol{u}_{\varepsilon}\right\|_{L^{p}(\Omega)}^{p} \leq C \varepsilon\|\boldsymbol{f}\|_{\mathbb{X}_{N}^{p}(\Omega)^{\prime}}^{p^{\prime}}
$$


Proof. It is clear that $E_{\varepsilon}$ is proper from (2.2), and that the functional $E_{\varepsilon}$ is strictly convex from Lemma 2.4. Moreover, $E_{\varepsilon}$ is lower semi-continuous on $\mathbb{K}_{\varphi}$ (cf. [5]). For any $\varepsilon \in(0,1]$ and for any $\boldsymbol{v} \in \mathbb{K}_{\varphi}$, it follows from (2.2) and the Young inequality that

$$
\begin{aligned}
E_{\varepsilon}[\boldsymbol{v}] & \geq \frac{\lambda}{p}\left\{\int_{\Omega}|\operatorname{curl} \boldsymbol{v}|^{p} d x+\frac{1}{\varepsilon} \int_{\Omega}|\operatorname{div} \boldsymbol{v}|^{p} d x\right\}-\|\boldsymbol{f}\|_{\mathbb{X}_{N}^{p}(\Omega)^{\prime}}\|\boldsymbol{v}\|_{\mathbb{X}_{N}^{p}(\Omega)} \\
& \geq \frac{\lambda}{p}\|\boldsymbol{v}\|_{\mathbb{X}_{N}^{p}(\Omega)}^{p}-C(\delta)\|\boldsymbol{f}\|_{\mathbb{X}_{N}^{p}(\Omega)^{\prime}}^{p^{\prime}}-\delta\|\boldsymbol{v}\|_{\mathbb{X}_{N}^{p}(\Omega)}^{p}
\end{aligned}
$$

for any $\delta>0$ and some $C(\delta)>0$. If we choose $\delta=\lambda /(2 p)$, then we have

$$
E_{\varepsilon}[\boldsymbol{v}] \geq \frac{\lambda}{2 p}\|\boldsymbol{v}\|_{\mathbb{X}_{N}^{p}(\Omega)}^{p}-C\left(\frac{\lambda}{2 p}\right)\|\boldsymbol{f}\|_{\mathbb{X}_{N}^{p}(\Omega)^{\prime}}^{p^{\prime}}
$$

Hence $E_{\varepsilon}$ is coercive on $\mathbb{K}_{\varphi}$. From Ekeland and Témam [8, Chapter II, Proposition 1.2], problem (3.3) has a unique minimizer $\boldsymbol{u}_{\varepsilon} \in \mathbb{K}_{\varphi}$.

For any $\boldsymbol{v} \in \mathbb{K}_{\varphi}$ and $0 \leq \mu \leq 1$, since $(1-\mu) \boldsymbol{u}_{\varepsilon}+\mu \boldsymbol{v}=\boldsymbol{u}_{\varepsilon}+\mu\left(\boldsymbol{v}-\boldsymbol{u}_{\varepsilon}\right) \in \mathbb{K}_{\varphi}$, we have

$$
\left.\frac{d}{d \mu} E_{\varepsilon}\left[\boldsymbol{u}_{\varepsilon}+\mu\left(\boldsymbol{v}-\boldsymbol{u}_{\varepsilon}\right)\right]\right|_{\mu=0} \geq 0 .
$$

That is to say, the minimizer $\boldsymbol{u}_{\varepsilon}$ satisfies the following inequality

$$
\begin{aligned}
& \int_{\Omega} S_{t}\left(x,\left|\operatorname{curl} \boldsymbol{u}_{\varepsilon}\right|^{2}\right) \operatorname{curl} \boldsymbol{u}_{\varepsilon} \cdot \operatorname{curl}\left(\boldsymbol{v}-\boldsymbol{u}_{\varepsilon}\right) d x \\
& +\frac{1}{\varepsilon} \int_{\Omega} S_{t}\left(x,\left(\operatorname{div} \boldsymbol{u}_{\varepsilon}\right)^{2}\right)\left(\operatorname{div} \boldsymbol{u}_{\varepsilon}\right) \operatorname{div}\left(\boldsymbol{v}-\boldsymbol{u}_{\varepsilon}\right) d x \\
& \quad \geq\left\langle\boldsymbol{f}, \boldsymbol{v}-\boldsymbol{u}_{\varepsilon}\right\rangle_{\mathbb{X}_{N}^{p}(\Omega)^{\prime}, \mathbb{X}_{N}^{p}(\Omega)} \text { for all } \boldsymbol{v} \in \mathbb{K}_{\varphi} .
\end{aligned}
$$

Taking $\boldsymbol{v}=\mathbf{0} \in \mathbb{K}_{\varphi}$ in (3.6) as a test function, we have

$$
\begin{aligned}
\lambda\left(\left\|\operatorname{curl} \boldsymbol{u}_{\varepsilon}\right\|_{\boldsymbol{L}^{p}(\Omega)}^{p}+\left\|\operatorname{div} \boldsymbol{u}_{\varepsilon}\right\|_{L^{p}(\Omega)}^{p}\right) \leq \lambda\left(\left\|\operatorname{curl} \boldsymbol{u}_{\varepsilon}\right\|_{L^{p}(\Omega)}^{p}+\frac{1}{\varepsilon}\left\|\operatorname{div} \boldsymbol{u}_{\varepsilon}\right\|_{L^{p}(\Omega)}^{p}\right) \\
\leq\left\langle\boldsymbol{f}, \boldsymbol{u}_{\varepsilon}\right\rangle_{\mathbb{X}_{N}^{p}(\Omega)^{\prime}, \mathbb{X}_{N}^{p}(\Omega)} \leq C(\delta)\|\boldsymbol{f}\|_{\mathbb{X}_{N}^{p}(\Omega)^{\prime}}^{p^{\prime}}+\delta\left\|\boldsymbol{u}_{\varepsilon}\right\|_{\mathbb{X}_{N}^{p}(\Omega)}^{p}
\end{aligned}
$$

for any $\delta>0$. If we choose $\delta>0$ so that $\delta<\lambda$, we have estimate (3.4). Using (3.4), we also get estimate (3.5).

Thus we showed that the variational problem (3.6) has a solution. We derive the uniqueness of solution to the problem (3.6).

Lemma 3.2. The variational inequality (3.6) has a unique solution. 
Proof. It suffices to prove the uniqueness. Let $\boldsymbol{u}_{\varepsilon}^{1}, \boldsymbol{u}_{\varepsilon}^{2} \in \mathbb{K}_{\varphi}$ be two solutions to (3.6). Then we have

$$
\begin{aligned}
& \int_{\Omega} S_{t}\left(x,\left|\operatorname{curl} \boldsymbol{u}_{\varepsilon}^{1}\right|^{2}\right) \operatorname{curl} \boldsymbol{u}_{\varepsilon}^{1} \cdot \operatorname{curl}\left(\boldsymbol{u}_{\varepsilon}^{2}-\boldsymbol{u}_{\varepsilon}^{1}\right) d x \\
& \qquad \frac{1}{\varepsilon} \int_{\Omega} S_{t}\left(x,\left(\operatorname{div} \boldsymbol{u}_{\varepsilon}^{1}\right)^{2}\right)\left(\operatorname{div} \boldsymbol{u}_{\varepsilon}^{1}\right) \operatorname{div}\left(\boldsymbol{u}_{\varepsilon}^{2}-\boldsymbol{u}_{\varepsilon}^{1}\right) d x \\
& \quad \geq\left\langle\boldsymbol{f}, \boldsymbol{u}_{\varepsilon}^{2}-\boldsymbol{u}_{\varepsilon}^{1}\right\rangle_{\mathbb{X}_{N}^{p}(\Omega)^{\prime}, \mathbb{X}_{N}^{p}(\Omega)}
\end{aligned}
$$

and

$$
\begin{aligned}
\int_{\Omega} S_{t}\left(x,\left|\operatorname{curl} \boldsymbol{u}_{\varepsilon}^{2}\right|^{2}\right) \operatorname{curl} \boldsymbol{u}_{\varepsilon}^{2} \cdot \operatorname{curl}\left(\boldsymbol{u}_{\varepsilon}^{1}-\boldsymbol{u}_{\varepsilon}^{2}\right) d x & \\
& +\frac{1}{\varepsilon} \int_{\Omega} S_{t}\left(x,\left(\operatorname{div} \boldsymbol{u}_{\varepsilon}^{2}\right)^{2}\right)\left(\operatorname{div} \boldsymbol{u}_{\varepsilon}^{2}\right) \operatorname{div}\left(\boldsymbol{u}_{\varepsilon}^{1}-\boldsymbol{u}_{\varepsilon}^{2}\right) d x \\
& \geq\left\langle\boldsymbol{f}, \boldsymbol{u}_{\varepsilon}^{1}-\boldsymbol{u}_{\varepsilon}^{2}\right\rangle_{\mathbb{X}_{N}^{p}(\Omega)^{\prime}, \mathbb{X}_{N}^{p}(\Omega)} .
\end{aligned}
$$

Hence we have

$$
\begin{aligned}
& \int_{\Omega}\left(S_{t}\left(x,\left|\operatorname{curl} \boldsymbol{u}_{\varepsilon}^{1}\right|^{2}\right) \operatorname{curl} \boldsymbol{u}_{\varepsilon}^{1}-S_{t}\left(x,\left|\operatorname{curl} \boldsymbol{u}_{\varepsilon}^{2}\right|^{2}\right) \operatorname{curl} \boldsymbol{u}_{\varepsilon}^{2}\right) \cdot \operatorname{curl}\left(\boldsymbol{u}_{\varepsilon}^{1}-\boldsymbol{u}_{\varepsilon}^{2}\right) d x \\
& +\frac{1}{\varepsilon} \int_{\Omega}\left(S_{t}\left(x,\left(\operatorname{div} \boldsymbol{u}_{\varepsilon}^{1}\right)^{2}\right)\left(\operatorname{div} \boldsymbol{u}_{\varepsilon}^{1}\right)-S_{t}\left(x,\left(\operatorname{div} \boldsymbol{u}_{\varepsilon}^{2}\right)\right)\left(\operatorname{div} \boldsymbol{u}_{\varepsilon}^{2}\right)\right) \operatorname{div}\left(\boldsymbol{u}_{\varepsilon}^{1}-\boldsymbol{u}_{\varepsilon}^{2}\right) d x \leq 0 .
\end{aligned}
$$

By Lemma 2.2, we can see that $\operatorname{curl}\left(\boldsymbol{u}_{\varepsilon}^{1}-\boldsymbol{u}_{\varepsilon}^{2}\right)=\mathbf{0}$ and $\operatorname{div}\left(\boldsymbol{u}_{\varepsilon}^{1}-\boldsymbol{u}_{\varepsilon}^{2}\right)=0$ in $\Omega$, so $\boldsymbol{u}_{\varepsilon}^{1}=\boldsymbol{u}_{\varepsilon}^{2}$.

Here we prepare the following lemma.

Lemma 3.3. For any $\psi \in L^{p}(\Omega)$, there exists $\boldsymbol{v}_{\psi} \in \mathbb{X}_{N}^{p}(\Omega)$ such that $\operatorname{curl} \boldsymbol{v}_{\psi}=$ $\mathbf{0}, \operatorname{div} \boldsymbol{v}_{\psi}=\psi$ in $\Omega$, and there exists a constant $C>0$ such that

$$
\left\|\boldsymbol{v}_{\psi}\right\|_{\mathbb{X}_{N}^{p}(\Omega)} \leq C\|\psi\|_{L^{p}(\Omega)}
$$

Thus $\boldsymbol{v}_{\psi} \in \mathbb{K}_{\varphi}$.

Proof. For any $\psi \in L^{p}(\Omega)$, the following Dirichlet problem

$$
\begin{cases}\Delta \phi=\psi & \text { in } \Omega \\ \phi=0 & \text { on } \Gamma\end{cases}
$$

has a unique solution $\phi \in W^{2, p}(\Omega) \cap W_{0}^{1, p}(\Omega)$. If we define $\boldsymbol{w}=\boldsymbol{\nabla} \phi$ in $\Omega$, then $\boldsymbol{w} \in \boldsymbol{W}^{1, p}(\Omega)$ satisfies $\operatorname{curl} \boldsymbol{w}=\mathbf{0}, \operatorname{div} \boldsymbol{w}=\psi$ in $\Omega$, Since $\boldsymbol{n} \times \nabla$ contains only the 
tangential derivatives, $\boldsymbol{n} \times \boldsymbol{w}=\boldsymbol{n} \times \nabla \phi=\mathbf{0}$ on $\Gamma$. Here let $\left\{\boldsymbol{e}_{1}, \ldots, \boldsymbol{e}_{I}\right\}$ be a basis of $\mathbb{K}_{N}^{p}(\Omega)$ such that $\left\langle\boldsymbol{n} \cdot \boldsymbol{e}_{i}, 1\right\rangle_{\Gamma_{k}}=\delta_{j k}$, and define

$$
\boldsymbol{v}_{\psi}=\boldsymbol{w}-\sum_{i=1}^{I}\langle\boldsymbol{w} \cdot \boldsymbol{n}, 1\rangle_{\Gamma_{i}} \boldsymbol{e}^{i}
$$

Then clearly $\left\langle\boldsymbol{v}_{\psi} \cdot \boldsymbol{n}, 1\right\rangle_{\Gamma_{k}}=0$ for $k=1, \ldots, I$. Hence $\boldsymbol{v}_{\psi} \in \mathbb{X}_{N}^{p}(\Omega)$ and $\boldsymbol{v}_{\psi} \in \mathbb{K}_{\varphi}$.

We are in a position to state one of the main theorems of this paper.

Theorem 3.4. Assume that $\boldsymbol{f} \in \mathbb{X}_{N}^{p}(\Omega)^{\prime}$. Then the variational inequality (3.1) has a unique solution $(\boldsymbol{u}, \pi) \in \widehat{\mathbb{K}}_{\varphi} \times L^{p^{\prime}}(\Omega)$, and there exists a constant $C>0$ depending only on $p, \lambda, \Lambda$ and $\Omega$ such that

$$
\|\boldsymbol{u}\|_{\mathbb{V}_{N}^{p}(\Omega)}^{p}+\|\pi\|_{L^{p^{\prime}(\Omega)}}^{p^{\prime}} \leq C\|\boldsymbol{f}\|_{\mathbb{X}_{N}^{p}(\Omega)^{\prime}}^{p^{\prime}}
$$

Proof. Let $\boldsymbol{u}_{\varepsilon}$ be a unique solution of (3.6). Then from (3.5), we can see that $\operatorname{div} \boldsymbol{u}_{\varepsilon} \rightarrow 0$ strongly in $L^{p}(\Omega)$ as $\varepsilon \rightarrow+0$. Define

$$
\pi_{\varepsilon}=-\frac{1}{\varepsilon} S_{t}\left(x,\left(\operatorname{div} \boldsymbol{u}_{\varepsilon}\right)^{2}\right) \operatorname{div} \boldsymbol{u}_{\varepsilon} .
$$

From (3.4), $\left\{\boldsymbol{u}_{\varepsilon}\right\}$ is bounded in $\mathbb{X}_{N}^{p}(\Omega)$. Passing to a subsequence, we can assume that $\boldsymbol{u}_{\varepsilon} \rightarrow \boldsymbol{u}$ weakly in $\mathbb{X}_{N}^{p}(\Omega)$ for some $\boldsymbol{u} \in \mathbb{X}_{N}^{p}(\Omega)$ and strongly in $\boldsymbol{L}^{p}(\Omega)$. Since $\operatorname{div} \boldsymbol{u}_{\varepsilon} \rightarrow \operatorname{div} \boldsymbol{u}$ in $\mathcal{D}^{\prime}(\Omega)$, we have $\operatorname{div} \boldsymbol{u}=0$ in $\Omega$. Hence $\boldsymbol{u} \in \mathbb{V}_{N}^{p}(\Omega)$. Since $\mathbb{K}_{\varphi}$ is weakly closed subset of $\mathbb{X}_{N}^{p}(\Omega)$, we have $\boldsymbol{u} \in \widehat{\mathbb{K}}_{\varphi}$, and from (3.4),

$$
\|\boldsymbol{u}\|_{\mathbb{V}_{N}^{p}(\Omega)}^{p}=\|\boldsymbol{u}\|_{\mathbb{X}_{N}^{p}(\Omega)}^{p} \leq \liminf _{\varepsilon \rightarrow+0}\left\|\boldsymbol{u}_{\varepsilon}\right\|_{\mathbb{X}_{N}^{p}(\Omega)}^{p} \leq C\|\boldsymbol{f}\|_{\mathbb{X}_{N}^{p}(\Omega)^{\prime}}^{p^{\prime}}
$$

We show that $\left\{\pi_{\varepsilon}\right\}_{\varepsilon \in(0,1]}$ is bounded in $L^{p^{\prime}}(\Omega)$. To show this we note that for any $\psi \in L^{p}(\Omega)$, there exists $\boldsymbol{v}_{\psi} \in \boldsymbol{W}^{1, p}(\Omega)$ as in Lemma 3.3. Taking $\boldsymbol{v}=M \boldsymbol{v}_{\psi}(M>0)$ as a test function in (3.6) and using (3.4), we have

$$
M \int_{\Omega} \pi_{\varepsilon} \psi d x \leq C\|\boldsymbol{f}\|_{\mathbb{X}_{N}^{p}(\Omega)^{\prime}}^{p^{\prime}}+M\|\boldsymbol{f}\|_{\mathbb{X}_{N}^{p}(\Omega)^{\prime}}\left\|\boldsymbol{v}_{\psi}\right\|_{\mathbb{X}_{N}^{p}(\Omega)} .
$$

If we divide this inequality by $M$ and letting $M \rightarrow \infty$, we can see that

$$
\int_{\Omega} \pi_{\varepsilon} \psi d x \leq C\|\boldsymbol{f}\|_{\mathbb{X}_{N}^{p}(\Omega)^{\prime}}\|\psi\|_{L^{p}(\Omega)} \text { for all } \psi \in L^{p}(\Omega) .
$$

This implies that

$$
\left|\int_{\Omega} \pi_{\varepsilon} \psi d x\right| \leq C\|\boldsymbol{f}\|_{\mathbb{X}_{N}^{p}(\Omega)^{\prime}}\|\psi\|_{L^{p}(\Omega)} \text { for all } \psi \in L^{p}(\Omega) .
$$


So, it follows that

$$
\left\|\pi_{\varepsilon}\right\|_{L^{p^{\prime}}(\Omega)} \leq C\|\boldsymbol{f}\|_{\mathbb{X}_{N}^{p}(\Omega)^{\prime}}
$$

Thus $\left\{\pi_{\varepsilon}\right\}$ is bounded in $L^{p^{\prime}}(\Omega)$. Passing to a subsequence, we can assume that $\pi_{\varepsilon} \rightarrow \pi$ weakly in $L^{p^{\prime}}(\Omega)$ for some $\pi \in L^{p^{\prime}}(\Omega)$ and

$$
\|\pi\|_{L^{p^{\prime}}(\Omega)}^{p^{\prime}} \leq \liminf _{\varepsilon \rightarrow+0}\left\|\pi_{\varepsilon}\right\|_{L^{p^{\prime}}(\Omega)}^{p^{\prime}} \leq C \Lambda\|\boldsymbol{f}\|_{\mathbb{X}_{N}^{p}(\Omega)^{\prime}}^{p^{\prime}}
$$

By the monotonicity of $S_{t}$, for any $\boldsymbol{v} \in \mathbb{K}_{\varphi}$,

$$
\begin{aligned}
\int_{\Omega} S_{t}\left(x,\left|\operatorname{curl} \boldsymbol{u}_{\varepsilon}\right|^{2}\right) \operatorname{curl} \boldsymbol{u}_{\varepsilon} \cdot \operatorname{curl}\left(\boldsymbol{u}_{\varepsilon}\right. & -\boldsymbol{v}) d x \\
& \geq \int_{\Omega} S_{t}\left(x,|\operatorname{curl} \boldsymbol{v}|^{2}\right) \operatorname{curl} \boldsymbol{v} \cdot \operatorname{curl}\left(\boldsymbol{u}_{\varepsilon}-\boldsymbol{v}\right) d x .
\end{aligned}
$$

Hence we have

$$
\begin{aligned}
\int_{\Omega} S_{t}\left(x,|\operatorname{curl} \boldsymbol{v}|^{2}\right) \operatorname{curl} \boldsymbol{v} \cdot \operatorname{curl}\left(\boldsymbol{v}-\boldsymbol{u}_{\varepsilon}\right) d x-\int_{\Omega} & \pi_{\varepsilon} \operatorname{div}\left(\boldsymbol{v}-\boldsymbol{u}_{\varepsilon}\right) d x \\
& \geq\left\langle\boldsymbol{f}, \boldsymbol{v}-\boldsymbol{u}_{\varepsilon}\right\rangle_{\mathbb{X}_{N}^{p}(\Omega)^{\prime}, \mathbb{X}_{N}^{p}(\Omega)}
\end{aligned}
$$

Since $\boldsymbol{u}_{\varepsilon} \rightarrow \boldsymbol{u}$ weakly in $\mathbb{X}_{N}^{p}(\Omega), \pi_{\varepsilon} \rightarrow \pi$ weakly in $L^{p^{\prime}}(\Omega)$ and div $\boldsymbol{u}_{\varepsilon} \rightarrow 0$ strongly in $L^{p}(\Omega)$, letting $\varepsilon \rightarrow+0$ in (3.10), we can derive

$$
\begin{aligned}
\int_{\Omega} S_{t}\left(x,|\operatorname{curl} \boldsymbol{v}|^{2}\right) \operatorname{curl} \boldsymbol{v} \cdot \operatorname{curl}(\boldsymbol{v}-\boldsymbol{u}) d x-\int_{\Omega} \pi \operatorname{div} \boldsymbol{v} d x \\
\qquad\langle\boldsymbol{f}, \boldsymbol{v}-\boldsymbol{u}\rangle_{\mathbb{X}_{N}^{p}(\Omega)^{\prime}, \mathbb{X}_{N}^{p}(\Omega)} \text { for all } \boldsymbol{v} \in \mathbb{K}_{\varphi}
\end{aligned}
$$

For any $\boldsymbol{w} \in \mathbb{K}_{\varphi}$, taking $\boldsymbol{v}=(1-\mu) \boldsymbol{u}+\mu \boldsymbol{w}=\boldsymbol{u}+\mu(\boldsymbol{w}-\boldsymbol{u}), 0<\mu<1$ as a test function of (3.11), we have

$$
\begin{array}{r}
\int_{\Omega} S_{t}\left(x,|\operatorname{curl}(\boldsymbol{u}+\mu(\boldsymbol{w}-\boldsymbol{u}))|^{2}\right) \operatorname{curl}(\boldsymbol{u}+\mu(\boldsymbol{w}-\boldsymbol{u})) \cdot \mu \operatorname{curl}(\boldsymbol{w}-\boldsymbol{u}) d x \\
-\mu \int_{\Omega} \pi \operatorname{div} \boldsymbol{w} d x \geq \mu\langle\boldsymbol{f}, \boldsymbol{w}-\boldsymbol{u}\rangle_{\mathbb{X}_{N}^{p}(\Omega)^{\prime}, \mathbb{X}_{N}^{p}(\Omega)} .
\end{array}
$$

If we divide both hand sides by $\mu$, and let $\mu \rightarrow+0$, then we have

$$
\begin{aligned}
\int_{\Omega} S_{t}\left(x,|\operatorname{curl} \boldsymbol{u}|^{2}\right) \operatorname{curl} \boldsymbol{u} \cdot \operatorname{curl}(\boldsymbol{w}-\boldsymbol{u}) d x-\int_{\Omega} \pi \operatorname{div} \boldsymbol{w} d x \\
\quad \geq\langle\boldsymbol{f}, \boldsymbol{w}-\boldsymbol{u}\rangle_{\mathbb{X}_{N}^{p}(\Omega)^{\prime}, \mathbb{X}_{N}^{p}(\Omega)} \text { for all } \boldsymbol{w} \in \mathbb{K}_{\varphi} .
\end{aligned}
$$

This means that the inequality (3.1) holds. 
Finally, we show the uniqueness of the solution. Let $\left(\boldsymbol{u}_{1}, \pi_{1}\right),\left(\boldsymbol{u}_{2}, \pi_{2}\right) \in \widehat{\mathbb{K}}_{\varphi} \times L^{p^{\prime}}(\Omega)$ be two solution of (3.1). Then since $\operatorname{div} \boldsymbol{u}_{i}=0$ in $\Omega$ for $i=1,2$, we have

$$
\int_{\Omega} S_{t}\left(x,\left|\operatorname{curl} \boldsymbol{u}_{i}\right|^{2}\right) \operatorname{curl} \boldsymbol{u}_{i} \cdot \operatorname{curl}\left(\boldsymbol{u}_{j}-\boldsymbol{u}_{i}\right) d x \geq\left\langle\boldsymbol{f}, \boldsymbol{u}_{j}-\boldsymbol{u}_{i}\right\rangle_{\mathbb{X}_{N}^{p}(\Omega)^{\prime}, \mathbb{X}_{N}^{p}(\Omega)} \text { for } i \neq j .
$$

Hence

$$
\int_{\Omega}\left(S_{t}\left(x,\left|\operatorname{curl} \boldsymbol{u}_{1}\right|^{2}\right) \operatorname{curl} \boldsymbol{u}_{1}-S_{t}\left(x,\left|\operatorname{curl} \boldsymbol{u}_{2}\right|^{2}\right) \operatorname{curl} \boldsymbol{u}_{2}\right) \cdot \operatorname{curl}\left(\boldsymbol{u}_{1}-\boldsymbol{u}_{2}\right) d x \leq 0 .
$$

By the monotonicity of $S_{t}$ (Lemma 2.2), we have curl $\boldsymbol{u}_{1}=\operatorname{curl} \boldsymbol{u}_{2}$ in $\Omega$, so $\boldsymbol{u}_{1}=\boldsymbol{u}_{2}$. Let $\boldsymbol{v} \in \mathbb{K}_{\varphi}$. From (3.12) with $\boldsymbol{w}=\boldsymbol{v}$ and $\boldsymbol{w}=-\boldsymbol{v}$, we have

$$
\begin{aligned}
\int_{\Omega} S_{t}\left(x,\left|\operatorname{curl} \boldsymbol{u}_{1}\right|^{2}\right) \operatorname{curl} \boldsymbol{u}_{1} \cdot \operatorname{curl}\left(\boldsymbol{v}-\boldsymbol{u}_{1}\right) d x-\int_{\Omega} \pi_{1} \operatorname{div} & \boldsymbol{v} d x \\
& \geq\left\langle\boldsymbol{f}, \boldsymbol{v}-\boldsymbol{u}_{1}\right\rangle_{\mathbb{X}_{N}^{p}(\Omega)^{\prime}, \mathbb{X}_{N}^{p}(\Omega)}
\end{aligned}
$$

and

$$
\begin{aligned}
\int_{\Omega} S_{t}\left(x,\left|\operatorname{curl} \boldsymbol{u}_{1}\right|^{2}\right) \operatorname{curl} \boldsymbol{u}_{1} \cdot \operatorname{curl}\left(-\boldsymbol{v}-\boldsymbol{u}_{1}\right) d x+\int_{\Omega} \pi_{2} \operatorname{div} \boldsymbol{v} d x & \\
& \geq\left\langle\boldsymbol{f},-\boldsymbol{v}-\boldsymbol{u}_{1}\right\rangle_{\mathbb{X}_{N}^{p}(\Omega)^{\prime}, \mathbb{X}_{N}^{p}(\Omega) .}
\end{aligned}
$$

Therefore, we have

$$
\begin{aligned}
\int_{\Omega}\left(\pi_{1}-\pi_{2}\right) \operatorname{div} \boldsymbol{v} d x \leq & 2\left\langle\boldsymbol{f}, \boldsymbol{u}_{1}\right\rangle_{\mathbb{X}_{N}^{p}(\Omega)^{\prime}, \mathbb{X}_{N}^{p}(\Omega)} \\
& -2 \int_{\Omega} S_{t}\left(x,\left|\operatorname{curl} \boldsymbol{u}_{1}\right|^{2}\right)\left|\operatorname{curl} \boldsymbol{u}_{1}\right|^{2} d x \text { for all } \boldsymbol{v} \in \mathbb{K}_{\varphi} .
\end{aligned}
$$

From (3.12) with $\boldsymbol{w}=\mathbf{0}$, we see that

$$
c:=\left\langle\boldsymbol{f}, \boldsymbol{u}_{1}\right\rangle_{\mathbb{X}_{N}^{p}(\Omega)^{\prime}, \mathbb{X}_{N}^{p}(\Omega)}-\int_{\Omega} S_{t}\left(x,\left|\operatorname{curl} \boldsymbol{u}_{1}\right|^{2}\right)\left|\operatorname{curl} \boldsymbol{u}_{1}\right|^{2} d x \geq 0
$$

For any $\psi \in C_{0}^{\infty}(\Omega)$, choose $\boldsymbol{v}_{\psi}$ as in Lemma 3.3. Then from (3.13), we have

$$
\int_{\Omega}\left(\pi_{1}-\pi_{2}\right) \operatorname{div} \boldsymbol{v}_{\psi} d x \leq 2 c .
$$

For large $M>0$, since $M \boldsymbol{v}_{\psi} \in \mathbb{K}_{\varphi}$, we see that

$$
\int_{\Omega}\left(\pi_{1}-\pi_{2}\right) \operatorname{div} \boldsymbol{v}_{\psi} d x \leq \frac{2 c}{M}
$$


Letting $M \rightarrow \infty$, we have

$$
\int_{\Omega}\left(\pi_{1}-\pi_{2}\right) \psi d x \leq 0 \text { for all } \psi \in C_{0}^{\infty}(\Omega)
$$

This implies that

$$
\int_{\Omega}\left(\pi_{1}-\pi_{2}\right) \psi d x=0 \text { for all } \psi \in C_{0}^{\infty}(\Omega) .
$$

By the celebrated Du Bois Raymond Lemma, we have $\pi_{1}=\pi_{2}$ a.e. in $\Omega$. This completes the proof of Theorem 3.4.

\section{CONTINUOUS DEPENDENCE ON THE DATA}

In this section, we show the continuous dependence of the solution obtained in section 3 to problem (3.1) on the data. Let $\boldsymbol{f} \in \mathbb{X}_{N}^{p}(\Omega)^{\prime}$ and $\varphi \in L^{\infty}(\Omega)$. For solution $\boldsymbol{u}$ of (3.1), we consider the following variational inequality.

$$
\begin{aligned}
\int_{\Omega} S_{t}\left(x,|\operatorname{curl} \boldsymbol{u}|^{2}\right) \operatorname{curl} \boldsymbol{u} \cdot \operatorname{curl}(\boldsymbol{v}-\boldsymbol{u}) d x \geq\langle\boldsymbol{f}, \boldsymbol{v}-\boldsymbol{u}\rangle_{\mathbb{X}_{N}^{p}(\Omega)^{\prime}, \mathbb{X}_{N}^{p}(\Omega)} \\
=\langle\boldsymbol{f}, \boldsymbol{v}-\boldsymbol{u}\rangle_{\mathbb{V}_{N}^{p}(\Omega)^{\prime}, \mathbb{V}_{N}^{p}(\Omega)}
\end{aligned}
$$

for all $v \in \widehat{\mathbb{K}}_{\varphi}$.

Lemma 4.1. If $(\boldsymbol{u}, \pi) \in \widehat{\mathbb{K}}_{\varphi} \times L^{p^{\prime}}(\Omega)$ is a unique solution of (3.1), then $\boldsymbol{u} \in \widehat{\mathbb{K}}_{\varphi}$ is a unique solution of (4.1).

Proof. It is clear that $\boldsymbol{u} \in \widehat{\mathbb{K}}_{\varphi}$ is a solution of (4.1), since $\boldsymbol{v} \in \widehat{\mathbb{K}}_{\varphi}$ satisfies $\operatorname{div} \boldsymbol{v}=0$ in $\Omega$. Let $\boldsymbol{u}, \widetilde{\boldsymbol{u}}$ be two solutions of (4.1). Then

$$
\int_{\Omega} S_{t}\left(x,|\operatorname{curl} \boldsymbol{u}|^{2}\right) \operatorname{curl} \boldsymbol{u} \cdot \operatorname{curl}(\widetilde{\boldsymbol{u}}-\boldsymbol{u}) d x \geq\langle\boldsymbol{f}, \widetilde{\boldsymbol{u}}-\boldsymbol{u}\rangle_{\mathbb{V}_{N}^{p}(\Omega)^{\prime}, \mathbb{V}_{N}^{p}(\Omega)}
$$

and

$$
\int_{\Omega} S_{t}\left(x,|\operatorname{curl} \widetilde{\boldsymbol{u}}|^{2}\right) \operatorname{curl} \widetilde{\boldsymbol{u}} \cdot \operatorname{curl}(\boldsymbol{u}-\widetilde{\boldsymbol{u}}) d x \geq\langle\boldsymbol{f}, \boldsymbol{u}-\widetilde{\boldsymbol{u}}\rangle_{\mathbb{V}_{N}^{p}(\Omega)^{\prime}, \mathbb{V}_{N}^{p}(\Omega)} .
$$

Therefore, we have

$$
\int_{\Omega}\left(S_{t}\left(x,|\operatorname{curl} \boldsymbol{u}|^{2}\right) \operatorname{curl} \boldsymbol{u}-S_{t}\left(x,|\operatorname{curl} \widetilde{\boldsymbol{u}}|^{2}\right) \operatorname{curl} \widetilde{\boldsymbol{u}}\right) \cdot \operatorname{curl}(\boldsymbol{u}-\widetilde{\boldsymbol{u}}) d x \leq 0 .
$$

By the monotonicity lemma (Lemma 2.2), we have $\boldsymbol{u}=\widetilde{\boldsymbol{u}}$ in $\mathbb{V}_{N}^{p}(\Omega)$.

Now, we give the second main theorem of this paper. 
Theorem 4.2. Assume that $F: \mathbb{R} \rightarrow[0, \infty)$ is a continuous function satisfying that there exists a constant $\nu>0$ such that $\nu \leq F(s)$ for all $s \in \mathbb{R}$. Let $\boldsymbol{f}_{n}, \boldsymbol{f} \in \mathbb{X}_{N}^{p}(\Omega)^{\prime}\left(\subset \mathbb{V}_{N}^{p}(\Omega)^{\prime}\right)$ and $\varphi_{n}, \varphi \in L^{\infty}(\Omega)$, and let $\left(\boldsymbol{u}_{n}, \pi_{n}\right) \in \widehat{\mathbb{K}}_{\varphi_{n}} \times L^{p^{\prime}}(\Omega)$ and $(\boldsymbol{u}, \pi) \in \widehat{\mathbb{K}}_{\varphi} \times L^{p^{\prime}}(\Omega)$ be unique solutions of (3.1) with $\varphi=\varphi_{n}$ and $\varphi=\varphi$, respectively. If $\boldsymbol{f}_{n} \rightarrow \boldsymbol{f}$ in $\mathbb{X}_{N}^{p}(\Omega)^{\prime}\left(\right.$ so in $\left.\mathbb{V}_{N}^{p}(\Omega)^{\prime}\right)$ and $\varphi_{n} \rightarrow \varphi$ in $L^{\infty}(\Omega)$ as $n \rightarrow \infty$, then $\boldsymbol{u}_{n} \rightarrow \boldsymbol{u}$ strongly in $\mathbb{V}_{N}^{p}(\Omega)$ and $\pi_{n} \rightarrow \pi$ weakly in $L^{p^{\prime}}(\Omega)$.

Proof. In order to show that $\boldsymbol{u}_{n} \rightarrow \boldsymbol{u}$ strongly in $\mathbb{V}_{N}^{p}(\Omega)$, we apply the result of Mosco [12, Theorem A]. Define an operator $S: \mathbb{V}_{N}^{p}(\Omega) \rightarrow \mathbb{V}_{N}^{p}(\Omega)^{\prime}$ by

$$
\langle S \boldsymbol{u}, \boldsymbol{v}\rangle=\int_{\Omega} S_{t}\left(x,|\operatorname{curl} \boldsymbol{u}|^{2}\right) \operatorname{curl} \boldsymbol{u} \cdot \operatorname{curl} \boldsymbol{v} d x \text { for } \boldsymbol{u}, \boldsymbol{v} \in \mathbb{V}_{N}^{p}(\Omega) .
$$

By the Hölder inequality, since

$$
\begin{aligned}
&\left|\int_{\Omega} S_{t}\left(x,|\operatorname{curl} \boldsymbol{u}|^{2}\right) \operatorname{curl} \boldsymbol{u} \cdot \operatorname{curl} \boldsymbol{v} d x\right| \\
& \leq\left(\int_{\Omega}\left|S_{t}\left(x,|\operatorname{curl} \boldsymbol{u}|^{2}\right) \operatorname{curl} \boldsymbol{u}\right|^{p^{\prime}} d x\right)^{1 / p^{\prime}}\left(\int_{\Omega}|\operatorname{curl} \boldsymbol{v}|^{p} d x\right)^{1 / p} \\
& \leq \Lambda\|\boldsymbol{u}\|_{\mathbb{V}_{N}^{p}(\Omega)}^{p-1}\|\boldsymbol{v}\|_{\mathbb{V}_{N}^{p}(\Omega)},
\end{aligned}
$$

the operator $S$ is well defined. Furthermore, define operators $T_{n}$ and $T$ from $\mathbb{V}_{N}^{p}(\Omega)$ to $\mathbb{V}_{N}^{p}(\Omega)^{\prime}$ by

$$
T_{n} \boldsymbol{u}=S \boldsymbol{u}-\boldsymbol{f}_{n} \text { and } T \boldsymbol{u}=S \boldsymbol{u}-\boldsymbol{f} .
$$

We check conditions I, II and III in Theorem A of Mosco [12] in the following lemmas.

First we check Mosco's condition I.

Lemma 4.3. The above operators $T_{n}$ and $T$ are monotone hemi-continuous mappings from $\mathbb{V}_{N}^{p}(\Omega)$ to $\mathbb{V}_{N}^{p}(\Omega)^{\prime}$, and $\left\{T_{n}\right\}$ is uniformly bounded in $\mathbb{V}_{N}^{p}(\Omega)$ and satisfies

$$
G(T) \subset s-\underline{\operatorname{Lim}} G\left(T_{n}\right) \text { in } \mathbb{V}_{N}^{p}(\Omega) \times \mathbb{V}_{N}^{p}(\Omega)^{\prime},
$$

where $G(T)$ and $G\left(T_{n}\right)$ denote the graphs of $T$ and $T_{n}$, respectively. Here we say that $\left\{T_{n}\right\}$ is uniformly bounded on $\mathbb{V}_{N}^{p}(\Omega)$, if for any bounded subset $B$ of $\mathbb{V}_{N}^{p}(\Omega)$, there exists a bounded subset $B^{\prime}$ of $\mathbb{V}_{N}^{p}(\Omega)^{\prime}$ such that $T_{n} B \subset B^{\prime}$ for each $n$.

Proof. That the operator $S$ is monotone follows from Lemma 2.2 and $S$ is clearly hemi-continuous since $S$ is a Carathéodory function. For any $\boldsymbol{v}, \boldsymbol{w} \in \mathbb{V}_{N}^{p}(\Omega)$, from (4.2),

$$
\begin{aligned}
\left|\left\langle T_{n} \boldsymbol{v}, \boldsymbol{w}\right\rangle_{\mathbb{V}_{N}^{p}(\Omega)^{\prime}, \mathbb{V}_{N}^{p}(\Omega)}\right|=\mid \int_{\Omega} S_{t}\left(x,|\operatorname{curl} \boldsymbol{v}|^{2}\right) \operatorname{curl} \boldsymbol{v} \cdot \operatorname{curl} \boldsymbol{w} d x \\
\quad-\left\langle\boldsymbol{f}_{n}, \boldsymbol{w}\right\rangle_{\mathbb{V}_{N}^{p}(\Omega)^{\prime}, \mathbb{V}_{N}^{p}(\Omega)} \mid \\
\leq \quad\left(\Lambda\|\boldsymbol{v}\|_{\mathbb{V}_{N}^{p}(\Omega)}^{p-1}+\left\|\boldsymbol{f}_{n}\right\|_{\mathbb{V}_{N}^{p}(\Omega)^{\prime}}\right)\|\boldsymbol{w}\|_{\mathbb{V}_{N}^{p}(\Omega)}
\end{aligned}
$$


Since $\boldsymbol{f}_{n} \rightarrow \boldsymbol{f}$ in $\mathbb{V}_{N}^{p}(\Omega)^{\prime}$, we can assume that there exists a constant $C_{0}>0$ such that $\left\|\boldsymbol{f}_{n}\right\|_{\mathbb{V}_{N}^{p}(\Omega)^{\prime}} \leq C_{0}$. Hence

$$
\left\|T_{n} \boldsymbol{v}\right\|_{\mathbb{V}_{N}^{p}(\Omega)^{\prime}} \leq \Lambda\|\boldsymbol{v}\|_{\mathbb{V}_{N}^{p}(\Omega)}^{p-1}+C_{0}
$$

Thus $\left\{T_{n}\right\}$ is uniformly bounded in $\mathbb{V}_{N}^{p}(\Omega)$. The inclusion (4.3) means that for every $\boldsymbol{v} \in \mathbb{V}_{N}^{p}(\Omega)$, there exists $\boldsymbol{v}_{n} \in \mathbb{V}_{N}^{p}(\Omega)$ such that $\boldsymbol{v}_{n} \rightarrow \boldsymbol{v}$ strongly in $\mathbb{V}_{N}^{p}(\Omega)$ and $T_{n} \boldsymbol{v}_{n} \rightarrow T \boldsymbol{v}$ strongly in $\mathbb{V}_{N}^{p}(\Omega)^{\prime}$. We show this. For every $\boldsymbol{v} \in \mathbb{V}_{N}^{p}(\Omega)$, let $\boldsymbol{v}_{n}=\boldsymbol{v}$. For any $\boldsymbol{w} \in \mathbb{V}_{N}^{p}(\Omega)$,

$$
\begin{aligned}
& \left|\left\langle T_{n} \boldsymbol{v}_{n}-T \boldsymbol{v}, \boldsymbol{w}\right\rangle_{\mathbb{V}_{N}^{p}(\Omega)^{\prime}, \mathbb{V}_{N}^{p}(\Omega)}\right| \\
& \quad=\left|\left\langle S \boldsymbol{v}_{n}-S \boldsymbol{v}, \boldsymbol{w}\right\rangle_{\mathbb{V}_{N}^{p}(\Omega)^{\prime}, \mathbb{V}_{N}^{p}(\Omega)}-\left\langle\boldsymbol{f}_{n}-\boldsymbol{f}, \boldsymbol{w}\right\rangle_{\mathbb{V}_{N}^{p}(\Omega)^{\prime}, \mathbb{V}_{N}^{p}(\Omega)}\right| \\
& \quad=\left|\left\langle\boldsymbol{f}_{n}-\boldsymbol{f}, \boldsymbol{w}\right\rangle_{\mathbb{V}_{N}^{p}(\Omega)^{\prime}, \mathbb{V}_{N}^{p}(\Omega)}\right| \\
& \quad \leq\left\|\boldsymbol{f}_{n}-\boldsymbol{f}\right\|_{\mathbb{V}_{N}^{p}(\Omega)^{\prime}}\|\boldsymbol{w}\|_{\mathbb{V}_{N}^{p}(\Omega)} .
\end{aligned}
$$

Thus it follows from the hypothesis of the Theorem that

$$
\left\|T_{n} \boldsymbol{v}_{n}-T \boldsymbol{v}\right\|_{\mathbb{V}_{N}^{p}(\Omega)^{\prime}} \leq\left\|\boldsymbol{f}_{n}-\boldsymbol{f}\right\|_{\mathbb{V}_{N}^{p}(\Omega)} \rightarrow 0 \text { as } n \rightarrow \infty
$$

This completes the proof of Lemma 4.3.

Next we check Mosco’s condition II.

Lemma 4.4. If $\varphi_{n} \rightarrow \varphi$ in $L^{\infty}(\Omega)$, then

$$
\widehat{\mathbb{K}}_{\varphi}=\operatorname{Lim} \widehat{\mathbb{K}}_{\varphi_{n}} \text { in the sense of Mosco. }
$$

This means that if $\boldsymbol{v}_{n} \in \widehat{\mathbb{K}}_{\varphi_{n}}$ and $\boldsymbol{v}_{n} \rightarrow \boldsymbol{v}$ weakly in $\mathbb{V}_{N}^{p}(\Omega)$, then $\boldsymbol{v} \in \widehat{\mathbb{K}}_{\varphi}$, and for given $\boldsymbol{v} \in \widehat{\mathbb{K}}_{\varphi}$, there exists $\boldsymbol{v}_{n} \in \widehat{\mathbb{K}}_{\varphi_{n}}$ such that $\boldsymbol{v}_{n} \rightarrow \boldsymbol{v}$ strongly in $\mathbb{V}_{N}^{p}(\Omega)$.

Proof. Let $\boldsymbol{v}_{n} \in \widehat{\mathbb{K}}_{\varphi_{n}}$ and $\boldsymbol{v}_{n} \rightarrow \boldsymbol{v}$ weakly in $\mathbb{V}_{N}^{p}(\Omega)$. Then curl $\boldsymbol{v}_{n} \rightarrow$ curl $\boldsymbol{v}$ weakly in $\boldsymbol{L}^{p}(\Omega)$ from Lemma 2.5. For any measurable subset $\omega \subset \Omega$,

$$
\int_{\omega}|\operatorname{curl} \boldsymbol{v}|^{p} d x \leq \liminf _{n \rightarrow \infty} \int_{\omega}\left|\operatorname{curl} \boldsymbol{v}_{n}\right|^{p} d x \leq \liminf _{n \rightarrow \infty} \int_{\omega} F\left(\varphi_{n}\right)^{p} d x=\int_{\omega} F(\varphi)^{p} d x .
$$

This implies that $|\operatorname{curl} \boldsymbol{v}| \leq F(\varphi)$ a.e. in $\Omega$, so $\boldsymbol{v} \in \widehat{\mathbb{K}}_{\varphi}$.

Next, put $\lambda_{n}=\left\|F\left(\varphi_{n}\right)-F(\varphi)\right\|_{L^{\infty}(\Omega)}$, then $\lambda_{n} \rightarrow 0$ as $n \rightarrow \infty$. For given $\boldsymbol{v} \in \widehat{\mathbb{K}}_{\varphi}$, define $\boldsymbol{v}_{n}=\boldsymbol{v} / \mu_{n}$ with $\mu_{n}=1+\lambda_{n} / \nu$. Then we have

$$
\left|\operatorname{curl} \boldsymbol{v}_{n}\right|=\frac{1}{\mu_{n}}|\operatorname{curl} \boldsymbol{v}| \leq \frac{1}{\mu_{n}} F(\varphi) \leq F\left(\varphi_{n}\right)
$$


since

$$
\mu_{n}=1+\frac{\left\|F\left(\varphi_{n}\right)-F(\varphi)\right\|_{L^{\infty}(\Omega)}}{\nu} \geq 1+\frac{F(\varphi)-F\left(\varphi_{n}\right)}{F\left(\varphi_{n}\right)}=\frac{F(\varphi)}{F\left(\varphi_{n}\right)} .
$$

Thus $\boldsymbol{v}_{n} \in \widehat{\mathbb{K}}_{\varphi_{n}}$ and

$$
\left\|\boldsymbol{v}_{n}-\boldsymbol{v}\right\|_{\mathbb{V}_{N}^{p}(\Omega)}^{p}=\int_{\Omega}\left|\operatorname{curl}\left(\boldsymbol{v}_{n}-\boldsymbol{v}\right)\right|^{p} d x=\left(1-\frac{1}{\mu_{n}}\right) \int_{\Omega}|\operatorname{curl} \boldsymbol{v}|^{p} d x \rightarrow 0
$$

as $n \rightarrow \infty$. This completes the proof of Lemma 4.4 .

Finally we check Mosco’s condition III.

Lemma 4.5. For any $\boldsymbol{w} \in \widehat{\mathbb{K}}_{\varphi}$, there exists a continuous strictly increasing function $\beta:[0, \infty] \rightarrow[0, \infty]$ with $\beta(0)=0$ such that

$$
\beta\left(\|\boldsymbol{v}-\boldsymbol{w}\|_{\mathbb{V}_{N}^{p}(\Omega)}\right) \leq \liminf _{n \rightarrow \infty}\left\langle T_{n} \boldsymbol{v}-T \boldsymbol{w}, \boldsymbol{v}-\boldsymbol{w}\right\rangle_{\mathbb{V}_{N}^{p}(\Omega)^{\prime}, \mathbb{V}_{N}^{p}(\Omega)}
$$

for all $\boldsymbol{v} \in \mathbb{V}_{N}^{p}(\Omega)$ uniformly as $\boldsymbol{v}$ varies in a bounded set.

Proof. It follows from Lemma 2.2 that

$$
\begin{aligned}
& \left\langle T_{n} \boldsymbol{v}-T \boldsymbol{w}, \boldsymbol{v}-\boldsymbol{w}\right\rangle_{\mathbb{V}_{N}^{p}(\Omega)^{\prime}, \mathbb{V}_{N}^{p}(\Omega)} \\
& =\langle S \boldsymbol{v}-S \boldsymbol{w}, \boldsymbol{v}-\boldsymbol{w}\rangle_{\mathbb{V}_{N}^{p}(\Omega)^{\prime}, \mathbb{V}_{N}^{p}(\Omega)}-\left\langle\boldsymbol{f}_{n}-\boldsymbol{f}, \boldsymbol{v}-\boldsymbol{w}\right\rangle_{\mathbb{V}_{N}^{p}(\Omega)^{\prime}, \mathbb{V}_{N}^{p}(\Omega)} \\
& \quad \geq\left\{\begin{array}{cc}
c \int_{\Omega}|\operatorname{curl}(\boldsymbol{v}-\boldsymbol{w})|^{p} d x-\left\|\boldsymbol{f}_{n}-\boldsymbol{f}\right\|_{\mathbb{V}_{N}^{p}(\Omega)^{\prime}}\|\boldsymbol{v}-\boldsymbol{w}\|_{\mathbb{V}_{N}^{p}(\Omega)} & \text { if } p \geq 2, \\
c \int_{\Omega}(|\operatorname{curl} \boldsymbol{v}|+|\operatorname{curl} \boldsymbol{w}|)^{p-2}|\operatorname{curl}(\boldsymbol{v}-\boldsymbol{w})|^{2} d x & \text { if } 1<p<2 . \\
-\left\|\boldsymbol{f}_{n}-\boldsymbol{f}\right\|_{\mathbb{V}_{N}^{p}(\Omega)^{\prime}}\|\boldsymbol{v}-\boldsymbol{w}\|_{\mathbb{V}_{N}^{p}(\Omega)}
\end{array}\right.
\end{aligned}
$$

When $p \geq 2$, using the Young inequality, for some constant $C>0$ we have

$$
\begin{aligned}
c \int_{\Omega}|\operatorname{curl}(\boldsymbol{v}-\boldsymbol{w})|^{p} d x-\left\|\boldsymbol{f}_{n}-\boldsymbol{f}\right\|_{\mathbb{V}_{N}^{p}(\Omega)^{\prime}} & \|\boldsymbol{v}-\boldsymbol{w}\|_{\mathbb{V}_{N}^{p}(\Omega)} \\
& \geq \frac{c}{2}\|\boldsymbol{v}-\boldsymbol{w}\|_{\mathbb{V}_{N}^{p}(\Omega)}^{p}-C\left\|\boldsymbol{f}_{n}-\boldsymbol{f}\right\|_{\mathbb{V}_{N}^{p}(\Omega)^{\prime}}^{p^{\prime}}
\end{aligned}
$$

When $1<p<2$, we recall the reverse Hölder inequality (cf. Sobolev [15, p. 8]). Let $0<s<1$ and $s^{\prime}=s /(s-1)$. If $F \in L^{s}(\Omega), F G \in L^{1}(\Omega)$ and $\int_{\Omega}|G(s)|^{s^{\prime}} d x<\infty$, then

$$
\left(\int_{\Omega}|F(s)|^{s} d x\right)^{1 / s} \leq \int_{\Omega}|F(x) G(x)| d x\left(\int_{\Omega}|G(x)|^{s^{\prime}} d x\right)^{-1 / s^{\prime}},
$$


and we apply it, with $s=p / 2$ (so $s^{\prime}=p /(p-2)$ ), $F=|\operatorname{curl}(\boldsymbol{v}-\boldsymbol{w})|^{2}, G=$ $(|\operatorname{curl} \boldsymbol{v}|+|\operatorname{curl} \boldsymbol{w}|)^{p-2}$, in $\widehat{\Omega}=\{x \in \Omega ;|\operatorname{curl} \boldsymbol{v}(x)|+|\operatorname{curl} \boldsymbol{w}(x)| \neq 0\}$. So,

$$
\begin{aligned}
& \left(\int_{\widehat{\Omega}}\left(|\operatorname{curl}(\boldsymbol{v}-\boldsymbol{w})|^{2}\right)^{p / 2} d x\right)^{2 / p} \\
\leq & \int_{\widehat{\Omega}}|\operatorname{curl}(\boldsymbol{v}-\boldsymbol{w})|^{2}(|\operatorname{curl} \boldsymbol{v}|+|\operatorname{curl} \boldsymbol{w}|)^{p-2} d x\left(\int_{\widehat{\Omega}}(|\operatorname{curl} \boldsymbol{v}|+|\operatorname{curl} \boldsymbol{w}|)^{p} d x\right)^{(2-p) / 2},
\end{aligned}
$$

and so,

$$
\begin{aligned}
\int_{\widehat{\Omega}} \mid \operatorname{curl} & \left.(\boldsymbol{v}-\boldsymbol{w})\right|^{2}(|\operatorname{curl} \boldsymbol{v}|+|\operatorname{curl} \boldsymbol{w}|)^{p-2} d x \\
& \geq\left(\int_{\widehat{\Omega}}\left(|\operatorname{curl}(\boldsymbol{v}-\boldsymbol{w})|^{2}\right)^{p / 2} d x\right)^{2 / p}\left(\int_{\widehat{\Omega}}(|\operatorname{curl} \boldsymbol{v}|+|\operatorname{curl} \boldsymbol{w}|)^{p} d x\right)^{(p-2) / 2} .
\end{aligned}
$$

Since $\boldsymbol{v}$ varies in a bounded set in $\mathbb{V}_{N}^{p}(\Omega)$, we can assume that $\int_{\Omega}|\operatorname{curl} \boldsymbol{v}|^{p} d x \leq C_{1}$, so there exists a constant $c_{2}>0$ depending only on $p$ and $\boldsymbol{w}$ such that

$$
\int_{\widehat{\Omega}}|\operatorname{curl}(\boldsymbol{v}-\boldsymbol{w})|^{2}(|\operatorname{curl} \boldsymbol{v}|+|\operatorname{curl} \boldsymbol{w}|)^{p-2} d x \geq c_{2}\|\boldsymbol{v}-\boldsymbol{w}\|_{\mathbb{V}_{N}^{p}(\Omega)}^{2} .
$$

Thus we have

$$
\begin{array}{rl}
c \int_{\Omega}(|\operatorname{curl} \boldsymbol{v}|+|\operatorname{curl} \boldsymbol{w}|)^{p-2}|\operatorname{curl}(\boldsymbol{v}-\boldsymbol{w})|^{2} & d x-\left\|\boldsymbol{f}_{n}-\boldsymbol{f}\right\|_{\mathbb{V}_{N}^{p}(\Omega)^{\prime}}\|\boldsymbol{v}-\boldsymbol{w}\|_{\mathbb{V}_{N}^{p}(\Omega)} \\
& \geq \frac{c}{2}\|\boldsymbol{v}-\boldsymbol{w}\|_{\mathbb{V}_{N}^{p}(\Omega)}^{2}-C\left\|\boldsymbol{f}_{n}-\boldsymbol{f}\right\|_{\mathbb{V}_{N}^{p}(\Omega)^{\prime}}^{2} .
\end{array}
$$

Therefore, we can derive

$$
\liminf _{n \rightarrow \infty}\left\langle T_{n} \boldsymbol{v}-T \boldsymbol{w}, \boldsymbol{v}-\boldsymbol{w}\right\rangle_{\mathbb{V}_{N}^{p}(\Omega)^{\prime}, \mathbb{V}_{N}^{p}(\Omega)} \geq \begin{cases}\frac{c}{2}\|\boldsymbol{v}-\boldsymbol{w}\|_{\mathbb{V}_{N}^{p}(\Omega)}^{p} & \text { if } p \geq 2, \\ \frac{c}{2}\|\boldsymbol{v}-\boldsymbol{w}\|_{\mathbb{V}_{N}^{p}(\Omega)}^{2} & \text { if } 1<p<2 .\end{cases}
$$

If we put $\beta(s)=\frac{c}{2} s^{p \vee 2}$, where $p \vee 2=\max \{p, 2\}$, the conclusion holds. This completes the proof of Lemma 4.5 .

We continue the proof of Theorem 4.2. From Lemma 4.3, 4.4 and 4.5, the hypotheses of $\left[12\right.$, Theorem A] hold, and we can conclude that $\boldsymbol{u}_{n} \rightarrow \boldsymbol{u}$ strongly in $\mathbb{V}_{N}^{p}(\Omega)$.

Finally, we show that $\pi_{n} \rightarrow \pi$ weakly in $L^{p^{\prime}}(\Omega)$.

Lemma 4.6. If $\boldsymbol{v}_{j} \rightarrow \boldsymbol{v}$ strongly in $\mathbb{V}_{N}^{p}(\Omega)$ as $j \rightarrow \infty$, then

$$
S_{t}\left(x,\left|\operatorname{curl} \boldsymbol{v}_{j}\right|^{2}\right) \operatorname{curl} \boldsymbol{v}_{j} \rightarrow S_{t}\left(x,|\operatorname{curl} \boldsymbol{v}|^{2}\right) \operatorname{curl} \boldsymbol{v}
$$

strongly in $\boldsymbol{L}^{p^{\prime}}(\Omega)$ as $j \rightarrow \infty$. 
Proof. From Lemma 2.3, we have

$$
\begin{aligned}
& \left|S_{t}\left(x,\left|\operatorname{curl} \boldsymbol{v}_{j}\right|^{2}\right) \operatorname{curl} \boldsymbol{v}_{j}-S_{t}\left(x,|\operatorname{curl} \boldsymbol{v}|^{2}\right) \operatorname{curl} \boldsymbol{v}\right|^{p^{\prime}} \\
& \quad \leq \begin{cases}C_{1}\left|\operatorname{curl} \boldsymbol{v}_{j}-\operatorname{curl} \boldsymbol{v}\right|^{p} & \text { if } 1<p<2, \\
C_{1}\left(\left|\operatorname{curl} \boldsymbol{v}_{j}\right|+|\operatorname{curl} \boldsymbol{v}|\right)^{(p-2) p^{\prime}}\left|\operatorname{curl} \boldsymbol{v}_{j}-\operatorname{curl} \boldsymbol{v}\right|^{p^{\prime}} & \text { if } p \geq 2 .\end{cases}
\end{aligned}
$$

When $1<p<2$, the conclusion is clear. When $p \geq 2$, using Hölder inequality, we have

$$
\begin{aligned}
& \int_{\Omega}\left|S_{t}\left(x,\left|\operatorname{curl} \boldsymbol{v}_{j}\right|^{2}\right) \operatorname{curl} \boldsymbol{v}_{j} \rightarrow S_{t}\left(x,|\operatorname{curl} \boldsymbol{v}|^{2}\right) \operatorname{curl} \boldsymbol{v}\right|^{p^{\prime}} d x \\
& \quad \leq\left(\int_{\Omega}\left(\left|\operatorname{curl} \boldsymbol{v}_{j}\right|+|\operatorname{curl} \boldsymbol{v}|\right)^{p} d x\right)^{\left(p-p^{\prime}\right) / p}\left(\int_{\Omega}\left|\operatorname{curl} \boldsymbol{v}_{j}-\operatorname{curl} \boldsymbol{v}\right|^{p} d x\right)^{p^{\prime} / p} \rightarrow 0
\end{aligned}
$$

as $j \rightarrow \infty$. Here we used the fact

$$
\int_{\Omega}\left|\operatorname{curl} \boldsymbol{v}_{j}\right|^{p} d x \leq C
$$

for some constant independent of $j$ since $\boldsymbol{v}_{j} \rightarrow \boldsymbol{v}$ strongly in $\mathbb{V}_{N}^{p}(\Omega)$. This completes the proof of Lemma 4.6.

We continue the proof of Theorem 4.2. For any $\psi \in L^{p}(\Omega)$, choose $\boldsymbol{v}_{\psi} \in \boldsymbol{W}^{1, p}(\Omega)$ as in Lemma 3.3. We note that $\boldsymbol{v}_{\psi} \in \mathbb{K}_{\varphi_{n}} \cap \mathbb{K}_{\varphi}$. If we choose $-\boldsymbol{v}_{\psi}$ as a test function of (3.1), then we have

$$
\begin{aligned}
\int_{\Omega} S_{t}\left(x,\left|\operatorname{curl} \boldsymbol{u}_{n}\right|^{2}\right) \operatorname{curl} \boldsymbol{u}_{n} \cdot \operatorname{curl}\left(-\boldsymbol{v}_{\psi}-\boldsymbol{u}_{n}\right) d x+ & \int_{\Omega} \pi_{n} \operatorname{div} \boldsymbol{v}_{\psi} d x \\
& \geq\left\langle\boldsymbol{f},-\boldsymbol{v}_{\psi}-\boldsymbol{u}_{n}\right\rangle_{\mathbb{X}_{N}^{p}(\Omega)^{\prime}, \mathbb{X}_{N}^{p}(\Omega) .}
\end{aligned}
$$

Adding this inequality and (3.1) with $\boldsymbol{v}=\boldsymbol{v}_{\psi}$, we have

$$
\begin{aligned}
\int_{\Omega} S_{t}\left(x,\left|\operatorname{curl} \boldsymbol{u}_{n}\right|^{2}\right) \operatorname{curl} \boldsymbol{u}_{n} \cdot \operatorname{curl}( & \left.-\boldsymbol{v}_{\psi}-\boldsymbol{u}_{n}\right) d x \\
+\int_{\Omega} S_{t}\left(x,|\operatorname{curl} \boldsymbol{u}|^{2}\right) \operatorname{curl} \boldsymbol{u} \cdot & \operatorname{curl}\left(\boldsymbol{v}_{\psi}-\boldsymbol{u}\right) d x+\int_{\Omega}\left(\pi_{n}-\pi\right) \operatorname{div} \boldsymbol{v}_{\psi} d x \\
& \geq-\left\langle\boldsymbol{f}, \boldsymbol{u}_{n}\right\rangle_{\mathbb{X}_{N}^{p}(\Omega)^{\prime}, \mathbb{X}_{N}^{p}(\Omega)}-\langle\boldsymbol{f}, \boldsymbol{u}\rangle_{\mathbb{X}_{N}^{p}(\Omega)^{\prime}, \mathbb{X}_{N}^{p}(\Omega)} .
\end{aligned}
$$

Taking the lower limit of this inequality and using Lemma 4.6,

$$
\begin{aligned}
\liminf _{n \rightarrow \infty} \int_{\Omega}\left(\pi_{n}-\right. & \pi) \operatorname{div} \boldsymbol{v}_{\psi} d x \\
& \geq-2\langle\boldsymbol{f}, \boldsymbol{u}\rangle_{\mathbb{X}_{N}^{p}(\Omega)^{\prime}, \mathbb{X}_{N}^{p}(\Omega)}-2 \int_{\Omega} S_{t}\left(x,|\operatorname{curl} \boldsymbol{u}|^{2}\right)|\operatorname{curl} \boldsymbol{u}|^{2} d x=-2 c_{1}
\end{aligned}
$$


where $c_{1} \geq 0$. For any $M>0$, since $M \boldsymbol{v}_{\psi} \in \mathbb{K}_{\varphi}$ and $-M \boldsymbol{v}_{\psi} \in \mathbb{K}_{\varphi}$, we have

$$
-\frac{2 c_{1}}{M} \leq \liminf _{n \rightarrow \infty} \int_{\Omega}\left(\pi_{n}-\pi\right) \operatorname{div} \boldsymbol{v}_{\psi} d x \leq \limsup _{n \rightarrow \infty} \int_{\Omega}\left(\pi_{n}-\pi\right) \operatorname{div} \boldsymbol{v}_{\psi} d x \leq \frac{2 c_{1}}{M} .
$$

Letting $M \rightarrow \infty$ and using $\operatorname{div} \boldsymbol{v}_{\psi}=\psi$ in $\Omega$, we have

$$
\lim _{n \rightarrow \infty} \int_{\Omega}\left(\pi_{n}-\pi\right) \psi d x=0 \text { for all } \psi \in L^{p}(\Omega) .
$$

Since $\left\|\pi_{n}\right\|_{L^{p^{\prime}}(\Omega)} \leq C\left\|\boldsymbol{f}_{n}\right\|_{\mathbb{X}_{N}^{p}(\Omega)^{\prime}} \leq C_{1}$, where $C_{1}$ is a constant independent of $n$ since $\boldsymbol{f}_{n} \rightarrow \boldsymbol{f}$ in $\mathbb{X}_{N}^{p}(\Omega)^{\prime}$. This completes the proof of Theorem 4.2.

\section{REFERENCES}

[1] C. Amrouche, N. H. Seloula: $L^{p}$-theory for vector potentials and Sobolev's inequality for vector fields. C. R. Acad. Sci. Paris, Ser. 1. 349 (2011), 529-534.

[2] C. Amrouche, N. H. Seloula: $L^{p}$-theory for vector potentials and Sobolev inequalities for vector fields: Application to the Stokes equations with pressure boundary conditions. Math. Models and Methods in Appl. Sci. 23(4) (2013), 37-92.

[3] J. Aramaki: Existence of weak solution for a class of abstract coupling system associated with stationary electromagnetic system, Taiwanese J. Math. 22(3) (2018), 741-765.

[4] J. Aramaki: Variational problem involving operator curl associated with $p$-curl system. Turkish J. Math. 42(3) (2018), 949-966.

[5] J. Aramaki: Existence and regularity of a weak solution to a class of systems in a multi-connected domain. J. Part. Diff. Eq. 32(1) (2019), 1-19.

[6] J. Aramaki: Existence of weak solutions to stationary and evolutionary Maxwell-Stokes type problems and the asymptotic behavior of the solution. Adv. Math. Sci. Appl. 28(1) (2019), 29-57.

[7] R. Dautray, J. L. Lions: Analyse Mathématique et Calcul Numérique pour les Sciences et les Technique, 3 and 7. Masson, (1988).

[8] I. Ekeland, R. T. Témam: Convex Analysis and Variational Problems. SIAM, Philadelphia (1976).

[9] V. Girault, P. A. Raviart: Finite Element Method for Navier-Stokes Equations. Springer Series SCM, (1986). 
[10] F. Miranda, J. F. Rodrigues, L. Santos: On a p-curl system arising in electromagnetism. Discrete and Cont. Dyn. Systems Ser. S 6(3) (2012), 605-629.

[11] F. Miranda, J. F. Rodrigues, L. Santos: A class of stationary nonlinear Maxwell systems. Math. Models Methods Appl. Sci. 19(10) (2009), 1883-1905.

[12] U. Mosco: Convergence of convex sets and of solutions of variational inequality, Advances in Math. 3 (1969), 510-585.

[13] X. - - B. Pan: Existence and regularity of solutions to quasilinear systems of Maxwell and Maxwell-Stokes type. Car. Var. 55 (2016), 143.

[14] L. Prigozhin: On the Bean critical-state model in superconductivity. Euro. J. Appl. Math. 7 (1996), 237-247.

[15] S. L. Sobolev: Applications of Functional Analysis in Mathematical Physics. Translation of Mathematical Monographs, 7, AMS, Providence, R. I. (1963).

[16] R. Temam: Navier-Stokes Equations. Theory and Analysis. North-Holland, Amsterdam (1985).

[17] H. -M. Yin, B. Li, J. Zou: A degenerate evolution system modeling Bean's critical-state type-II superconductors. Discrete Contin. Dyn. Syst. 8 (2002), 781-794. 